\title{
Evolution of a shock generated by an impulsively accelerated, sinusoidal piston
}

\author{
N. Shen ${ }^{1}$, D. I. Pullin ${ }^{1}$, R. Samtaney ${ }^{2}$, and V. Wheatley ${ }^{3}$ \\ ${ }^{1}$ Graduate Aerospace Laboratories, California Institute of Technology, Pasadena, CA 91125, \\ USA \\ ${ }^{2}$ Mechanical Engineering, Physical Science and Engineering Division, King Abdullah \\ University of Science and Technology, Thuwal 23955-6900, Saudi Arabia \\ ${ }^{3}$ School of Mechanical and Mining Engineering, The University of Queensland, St Lucia, QLD \\ 4072, Australia
}

(Received xx; revised xx; accepted $\mathrm{xx}$ )

We consider the evolution of shock-wave generated by an impulsively accelerated, twodimensional almost planar piston with a sinusoidally corrugated surface of amplitude $\epsilon$. We develop a complex-variable formulation for a nonlinear theory of generalised geometrical shock dynamics (GGSD) (Best 1991, 1993) as a hierarchical expansion of the Euler equations that can be closed at any order. The zeroth-order truncation of GGSD is related to the equations of Whitham's geometrical shock dynamics (GSD) while higherorder corrections incorporate non-uniformity of the flow immediately behind the pistondriven shock. Numerical solutions to GGSD systems up to second order are coupled to an edge-detection algorithm in order to investigate the hypothesized development of a shock-shape curvature singularity as the rippled shock evolves. This singular behavior, together with the simultaneous development of a Mach-number discontinuity, is found at all orders of the GGSD hierarchy for both weak and strong shocks. The critical time at which a curvature singularity occurs converges as the order of the GGSD system increases at fixed $\epsilon$, and follows a scaling inversely proportional to $\epsilon$ at sufficiently small values. This result agrees with the weakly nonlinear GSD analysis of Mostert et al. (2018a) for a general Mach number perturbation on a planar shock, and suggests that this represents the universal behavior of a slightly perturbed, planar shock.

\section{Introduction}

The dynamics of shock-waves generated by the steady motion of a two-dimensional planar piston with a corrugated surface has been a classical hydrodynamic problem spanning over sixty years from the original work of Freeman (1955) to the more recent analysis of Bates (2015). The focus here concerns the stability of a rippled shock, which can be dynamically relevant to astrophysical phenomena (Maxwell et al. 1985; Drake et al. 1998), inertial confinement fusion applications (Matsui et al. 1999; Lindl et al. 2014), Bose-Einstein condensates and nonlinear optics (Hoefer et al. 2008). It has long been known that planar gaseous shock waves are linearly stable, that is, small perturbations along the shock surface decay over time following a power law (Lighthill 1949, 1950; Freeman 1955, 1957; Zaidel 1967; Van Moorhem \& George 1975). The predictions of linear analysis are supported by the pioneering experiments of Lapworth (1959) and Briscoe \& Kovtiz (1968). But the experiments do display noticeable singular flow behaviour associated with the formation of a triple point, or Mach stem on the shock front that cannot be explained by linear theory.

The dynamics of a triple point on a strongly perturbed shock was modeled by Whitham 
(1957), using his non-linear theory of geometrical shock dynamics (GSD), as a shock on a shock, or a "shock-shock" that propagates transversely along the shock profile. The cellular structure of Mach stems ubiquitously observed in detonation waves (Strehlow \& Fernandes 1965; Courant \& Friedrichs 1999) have motivated a number of theoretical investigations of the spontaneous formation of triple points (Majda \& Rosales 1983; Clavin \& Denet 2002; Faria et al. 2015). Shock instabilities are also reported in nonreacting flows associated with perturbations of various origin. The nonlinear analysis of Clavin (2013) shows the formation of a singularity in the slope of a winkled shock produced by a shock-vortex interaction, under the distinguished limit where the shock Mach number becomes infinite while the specific heat ratio of the gas approaches unity. A two-stage description of the shock evolution and an order-of-magnitude estimate for the Mach stem formation time was provided. Later, the shock tube experiment of Denet et al. (2015), and the direct numerical simulations of Lodato et al. (2016; 2017) confirmed the triple point formation of shock reflected off a wavy wall, but did not relate the formation time to the initial perturbation size of the wall. Wan et al. (2017) investigated numerically and experimentally the triple point trajectory due to shock interaction with a water wedge. Mostert et al. $(2018 a ; 2018 b)$ hypothesised a sinusoidal perturbation in the Mach number distribution for both initially flat and cylindrical shock geometry, finding that a shock curvature singularity, as a prelude to the formation of a triple point, occurs at a critical time $\tau_{c}$ that is inversely proportional to the initial perturbation amplitude, $\epsilon$. This result is obtained analytically via weakly nonlinear Fourier analysis using Whitham's GSD approximation.

The strict GSD framework of Mostert et al. (2018a) first neglects the non-uniform effects of flow behind the shock carried by the $C^{+}$characteristics. Second, the physical origin of the shock perturbation is not well defined owing to the restriction posed by the $A-M$ relation [see our Eq. (2.9)] on the initial conditions of the shock shape and Mach number profiles. In the present study, we address these issues by applying the generalised geometrical shock dynamics (GGSD) formulation, developed by Best (1991; 1993) as a hierarchical expansion of the Euler equations that can be closed at any order. The GGSD systems relaxes the $A-M$ relation while specifically incorporating the nonuniformity of the flow immediately behind the shock into its dynamical description in terms of evolution equations for higher order derivatives of the primitive flow variables. Katko et al. (2020) showed that, owing to the inclusion of flow non-uniformity, the GGSD model successfully captures the analytical solution for the expansion of a blast wave in comparison with the GSD result.

Adopting the GGSD theory, we analyse the specific problem of a shock generated by a two-dimensional impulsive piston with a sinusoidally corrugated surface, in complex coordinates. The linear stability of a piston-driven shock was also investigated by Bates (2012; 2015), who found an unstable region of the D'yakov parameter (D'yakov 1954) for gases of arbitrary equation-of-state, where a small perturbation on the shock front grows in time. Presently we focus on an ideal gas equation-of-state and propose a perturbation ansatz of a different nature where unstable modes are controlled by the intrinsic shock nonlinearity that ultimately leads to the spontaneous formation of a curvature singularity, detected numerically as a discontinuity of the Mach number distribution along the shock. The critical time at which singularity occurs converges as the order of the GGSD system increases from zero to two, and the inverse power law, $\tau_{c} \propto 1 / \epsilon$, established by Mostert et al. (2018a) holds universally true with the present inclusion of the non-uniform effects of the shocked flow.

The remainder of this paper is organized as follows. We first discuss in Section 2 the approximation error of GSD compared to the two-dimensional Euler equations, and 
introduce the GGSD expansion that improves the GSD theory. The analysis parallels Best (1993) but utilizes a different definition of the shock velocity. Evolution of a periodically perturbed planar shock is formulated in Section 3 using complex variables. The zeroth, first and second order truncated GGSD models are derived explicitly in the strong and weak shock limits as closed systems of partial differential equations that can be solved numerically using simple technique such as method-of-lines. A linear stability analysis is also given in Section 3. We then specialise in Section 4 to the motion of a shock generated by a corrugated piston of sinusoidal surface being impulsively accelerated to a steady velocity. The necessary initial conditions required to integrate the GGSD systems for this shock are derived. These require shock-jump conditions for gradients of physical quantities. Section 5 presents numerical results that characterise the shock shape curvature singularity formation, and demonstrate the inverse power law that predicts the onset of triple points. Finally, conclusions are drawn in Section 6.

\section{Generalized geometrical shock dynamics}

We begin by reviewing the GSD framework (Whitham 1957). Its limitations are identified in a direct comparison against the two-dimensional Euler equations. We then introduce GGSD developed by Best (1991), in order to improve the approximation error.

\subsection{Quasi-one-dimensional Euler equations}

The foundation for constructing the evolution equations for shocks in two-dimensional unsteady flow is the quasi-one-dimensional (1D) Euler equations that describe flow of a polytropic gas down a tube of slowly varying cross-sectional area $A=A(x)$, along the propagation direction denoted by the spatial variable $x$. By averaging the inviscid compressible flow equations across the tube, one obtains the following approximation (Whitham 1957)

$$
\begin{aligned}
& \rho_{t}+u \rho_{x}+\rho u_{x}+\rho u \frac{A^{\prime}(x)}{A(x)}=0, \\
& \rho\left(u_{t}+u u_{x}\right)+p_{x}=0 \\
& p_{t}+u p_{x}-a^{2}\left(\rho_{t}+u \rho_{x}\right)=0,
\end{aligned}
$$

where $\rho, u, p$ are fluid density, velocity and pressure, respectively; $a=\sqrt{\gamma p / \rho}$ is the local sound speed for a perfect-gas equation of state, $\gamma$ is the constant specific heat ratio; $x$, $t$ are distance and time; the prime symbol denotes full derivative with respect to $x$, and the subscript $t$ and $x$ are used to signal their respective partial derivatives. Equations (2.1) can be written in characteristic form as

$$
\begin{aligned}
& p_{t}+(u+a) p_{x}+\rho a\left(u_{t}+(u+a) u_{x}\right)+\rho a^{2} u A^{\prime} / A=0, \quad \text { on } \quad C^{+}: \frac{d x}{d t}=u+a, \\
& p_{t}+(u-a) p_{x}-\rho a\left(u_{t}+(u-a) u_{x}\right)+\rho a^{2} u A^{\prime} / A=0, \quad \text { on } \quad C^{-}: \frac{d x}{d t}=u-a, \\
& p_{t}+u p_{x}-a^{2}\left(\rho_{t}+u \rho_{x}\right)=0, \quad \text { on } \quad S: \frac{d x}{d t}=u .
\end{aligned}
$$

Denoting the trajectory of a shock propagating down the tube of uniform medium at rest by $x_{s}=x_{s}(t)$, its velocity is given by

$$
\frac{d x_{s}}{d t}=a_{0} M(t)
$$


where $a_{0}$ is the upstream speed-of-sound, $M$ is the shock Mach number. We define total time derivatives of $\rho, u$ and $p$ immediately behind the shock as their rate-of-change observed by a marker traveling with the shock, given by

$$
\frac{D \rho}{D t}=\rho_{t}+a_{0} M \rho_{x}, \quad \frac{D u}{D t}=u_{t}+a_{0} M u_{x}, \quad \frac{D p}{D t}=p_{t}+a_{0} M p_{x},
$$

The following Rankine-Hugoniet $(\mathrm{RH})$ conditions connect flow variables behind the shock to their upstream values via Mach number,

$$
\begin{aligned}
& u=F^{u}(M) \equiv \frac{2 a_{0}}{\gamma+1}\left(M-\frac{1}{M}\right), \\
& \rho=F^{\rho}(M) \equiv \frac{\rho_{0}(\gamma+1) M^{2}}{(\gamma-1) M^{2}+2}, \\
& p=F^{p}(M) \equiv \frac{\rho_{0} a_{0}^{2}}{\gamma(\gamma+1)}\left(2 \gamma M^{2}-\gamma+1\right), \\
& a=F^{a}(M) \equiv \frac{a_{0} \mu\left(2 \gamma M^{2}-\gamma+1\right)}{(\gamma+1) M},
\end{aligned}
$$

where $\rho_{0}$ is the upstream fluid density, and

$$
\mu=\sqrt{\frac{(\gamma-1) M^{2}+2}{2 \gamma M^{2}-\gamma+1}},
$$

is the post-shock Mach number.

\subsubsection{A-M relation}

By applying the $\mathrm{RH}$ conditions along the $C^{+}$characteristics instead of the shock, namely, substituting Eqs. (2.5) into Eq. (2.2a), Whitham (1957; 2011) obtained an expression for $M(A)$ that relates the shock Mach number, $M$, to the tube's local crosssectional area, $A$. This so-called $A-M$ relation gives the basis of the GSD theory. Here we attempt to recover the $A$ - $M$ relation using a different method. With (2.1) and (2.4) combined, all six partial derivatives, $p_{t}, p_{x}, u_{t}, u_{x}, \rho_{t}, \rho_{x}$, can be solved from the resulting six linear equations. In particular, we have

$$
\begin{aligned}
& p_{t}=\frac{a_{0} M \rho u a^{2}\left(a_{0} M-u\right) A^{\prime}+A\left[a^{2}\left(a_{0} M \rho \frac{D \rho}{D t}+\frac{D p}{D t}\right)+u \frac{D p}{D t}\left(a_{0} M-u\right)\right]}{A\left[a^{2}-\left(u-a_{0} M\right)^{2}\right]}, \\
& u_{t}=\frac{a_{0} M \rho u a^{2} A^{\prime}+A\left[\rho \frac{D u}{D t}\left(a^{2}+u\left(a_{0} M-u\right)\right)+a_{0} M \frac{D p}{D t}\right]}{A \rho\left[a^{2}-\left(u-a_{0} M\right)^{2}\right]} .
\end{aligned}
$$

Substituting (2.7) into the expression $\left(p_{t}+\rho a u_{t}\right)$ and using the identity $D / D t=$ $(d M / d t)(d / d M)$ then gives an equation from which $d M / d t$ can be solved, producing

$$
\left(\frac{d p}{d M}+\rho a \frac{d u}{d M}\right) \frac{d M}{d t}=-\left[\frac{a_{0} M \rho a^{2} u A^{\prime}}{(a+u) A}+\left(\frac{a_{0} M}{a+u}-1\right)\left(p_{t}+\rho a u_{t}\right)\right],
$$

where $d p / d M, d u / d M, \rho, a$ and $u$ are known as functions of $M$, from Eqs. (2.5).

In Whitham's (1957) development of the $A$ - $M$ relation, it is argued that $\left|p_{t}+\rho a u_{t}\right|$ must be small to justify the use "characteristic rule". In the current formulation, this is equivalent to neglecting the last term in (2.8), noting that the component $\left|a_{0} M /(a+u)-1\right|$ is uniformly bounded for all $M \in(1, \infty)$. As a result, using $d A / d x=(d A / d t) /\left(a_{0} M\right)$, 


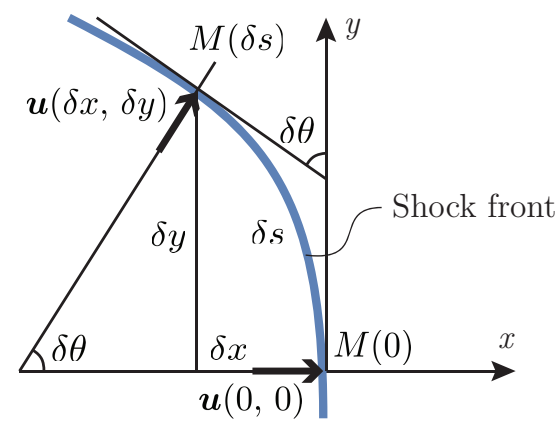

Figure 1. (a) Local Cartesian coordinates fixed at a point on shock normal ( $x$-direction) and tangential ( $y$-direction) to the curve. Downstream velocity vectors and Mach numbers are shown at the origin and a differential nearby point at $(\delta x, \delta y)$, whose arclength is $\delta s$.

the $A-M$ relation follows,

$$
\frac{1}{A} \frac{d A}{d M}=\frac{-M}{M^{2}-1}\left(1+\frac{2\left(1-\mu^{2}\right)}{(\gamma+1) \mu}\right)\left(1+2 \mu+\frac{1}{M^{2}}\right),
$$

which can then be integrated to give $M=M(A)$, up to a multiplying constant.

One useful feature of the present derivation of (2.9) is that, compared to Whitham's original "characteristic rule" $(1957 ; 2011)$, which essentially assumes that the nonuniform flow effect behind the shock carried by the $C^{+}$characteristics does not modify the shock motion, the error associated with this assumption can be exactly quantified. Here, the neglected term is the product of two terms, where $\left|a_{0} M /(a+u)-1\right|$, as a function of $M$ only, measures the geometrical closeness between the $C^{+}$characteristics and the shock, while $\left|p_{t}+\rho a u_{t}\right|$ captures the non-uniformity of the flow immediately behind the shock.

\subsection{Two-dimensional Euler equations}

We can extend the Quasi-1D theory to two-dimensional (2D) flows. A 2D shock-shape profile must be specified in terms of an intrinsic coordinate that labels points along the shock. Following Whitham's $(1957 ; 2011)$ GSD theory, we spatially parameterize points on the shock with the parameter $\beta$. This is defined as a continuous, monotonically increasing variable along the shock such that a point with fixed $\beta$ is instantaneously moving with velocity (in the laboratory frame) that is normal to the shock. This can be taken as the present definition of the "shock velocity". Conceptually it is related to the quasi-one-dimensional concept that the shock moves along "ray tubes" that are locally normal to the shock front.

For a given $\beta$ at time $t$, we set up a laboratory-fixed, local Cartesian coordinate system shown in figure 1 , whose origin sits on the shock point defined by $\beta$. The positive $x$ direction aligns with the instantaneous shock velocity at the origin and the positive $y$-direction corresponds to an increase of $\beta$ along the shock. The Mach number profile along the shock may be parameterized as a function of arclength, $s$, measured from the origin in the positive $y$-direction. In GSD, $s$ and $\beta$ are related by defining the normalized ray tube area $A$ as,

$$
A=\frac{d s}{d \beta}
$$


The flow that defines the shock is governed by the 2D Euler equations,

$$
\begin{aligned}
& \rho_{t}+u \rho_{x}+v \rho_{y}+\rho\left(u_{x}+v_{y}\right)=0, \\
& \rho\left(u_{t}+u u_{x}+v u_{y}\right)+p_{x}=0 \\
& \rho\left(v_{t}+u v_{x}+v v_{y}\right)+p_{y}=0 \\
& p_{t}+u p_{x}+v p_{y}+\gamma p\left(u_{x}+v_{y}\right)=0 .
\end{aligned}
$$

\subsubsection{Tangential derivatives}

In order to obtain an evolution equation analogous to (2.8) that governs the shock motion in 2D, all partial derivatives of the flow variables present in (2.11) must be evaluated immediately behind the shock. In particular, this can be done explicitly for the tangential derivatives, i.e., those with respect to $y$. At the origin, we have just downstream the shock, $x$ and $y$ components of flow velocity (in the laboratory frame), density and pressure given by

$$
\begin{aligned}
& u(0,0)=F^{u}(M(0)), \quad v(0,0)=0, \\
& \rho(0,0)=F^{\rho}(M(0)), \quad p(0,0)=F^{p}(M(0)) \text {. }
\end{aligned}
$$

At a nearby point on the shock $(\delta x, \delta y)$ with arclength $\delta s=\sqrt{\delta x^{2}+\delta y^{2}}$, making a tangent angle of $\delta \theta$ with the $y$-axis (figure 1(a)), these flow quantities become

$$
\begin{array}{lll}
u(\delta x, \delta y)=F^{u}(M(\delta s)) \cos (\delta \theta), & v(\delta x, \delta y)=F^{u}(M(\delta s)) \sin (\delta \theta), \\
\rho(\delta x, \delta y)=F^{\rho}(M(\delta s)), & & p(\delta x, \delta y)=F^{p}(M(\delta s)),
\end{array}
$$

where the Mach number has the Taylor expansion

$$
M(\delta s)=M(0)+\left.\frac{d M}{d s}\right|_{(0,0)} \delta s+o(\delta s) .
$$

To proceed we make use of the following three limits

$$
\lim _{\delta y \rightarrow 0} \delta x / \delta y=0, \quad \lim _{\delta y \rightarrow 0} \delta s / \delta y=1, \quad \lim _{\delta y \rightarrow 0} \delta \theta / \delta y=\kappa,
$$

where $\kappa$ is the signed curvature of the shock at the origin. As a result, Eqs. (2.13) are further Taylor expanded as,

$$
\begin{aligned}
& u(0,0)+\left.\frac{\partial u}{\partial y}\right|_{(0,0)} \delta y=F^{u}(M(0))+\left[\frac{d F^{u}}{d M} \frac{d M}{d s}\right]_{(0,0)} \delta y+o(\delta y), \\
& v(0,0)+\left.\frac{\partial v}{\partial y}\right|_{(0,0)} \delta y=F^{u}(M(0)) \delta \theta+o(\delta y), \\
& \rho(0,0)+\left.\frac{\partial \rho}{\partial y}\right|_{(0,0)} \delta y=F^{\rho}(M(0))+\left[\frac{d F^{\rho}}{d M} \frac{d M}{d s}\right]_{(0,0)} \delta y+o(\delta y), \\
& p(0,0)+\left.\frac{\partial p}{\partial y}\right|_{(0,0)} \delta y=F^{p}(M(0))+\left[\frac{d F^{p}}{d M} \frac{d M}{d s}\right]_{(0,0)} \delta y+o(\delta y) .
\end{aligned}
$$

Substituting (2.12) into (2.16) and applying the $\delta y \rightarrow 0$ limit then gives the desired 
tangential derivatives at the origin,

$$
\begin{aligned}
\frac{\partial u}{\partial y} & =\frac{d F^{u}}{d M} \frac{d M}{d s}, & \frac{\partial v}{\partial y} & =F^{u}(M) \kappa, \\
\frac{\partial \rho}{\partial y} & =\frac{d F^{\rho}}{d M} \frac{d M}{d s}, & \frac{\partial p}{\partial y} & =\frac{d F^{p}}{d M} \frac{d M}{d s},
\end{aligned}
$$

where we may write

$$
\frac{d M}{d s}=\frac{d M}{d \beta} \frac{d \beta}{d s}=\frac{1}{A} \frac{d M}{d \beta} .
$$

Using (2.10) and (2.18), the derivatives in (2.17) can be calculated at time $t$ provided both the shock shape and Mach number profiles are known functions of $\beta$.

\subsubsection{Time and normal derivatives}

Since the shock marker located at the origin at time $t$ has velocity normal to itself, the time and normal derivatives of flow variables immediately behind the shock are related through the total derivatives,

$$
\begin{aligned}
& \frac{D u}{D t}=\frac{d F^{u}}{d M}\left(\frac{\partial M}{\partial t}\right)_{\beta}=\frac{\partial u}{\partial t}+a_{0} M \frac{\partial u}{\partial x}, \\
& \frac{D v}{D t}=0=\frac{\partial v}{\partial t}+a_{0} M \frac{\partial v}{\partial x} \\
& \frac{D \rho}{D t}=\frac{d F^{\rho}}{d M}\left(\frac{\partial M}{\partial t}\right)_{\beta}=\frac{\partial \rho}{\partial t}+a_{0} M \frac{\partial \rho}{\partial x} \\
& \frac{D p}{D t}=\frac{d F^{p}}{d M}\left(\frac{\partial M}{\partial t}\right)_{\beta}=\frac{\partial p}{\partial t}+a_{0} M \frac{\partial p}{\partial x} .
\end{aligned}
$$

In addition, all the partial derivatives discussed so far satisfy the 2D Euler equations (2.11). Therefore, substituting (2.12) and (2.17) into (2.11), together with (2.19) gives eight linear equations for eight unknowns, i.e., the $x$ and $t$ partial derivatives of $u, v, \rho$ and $p$. This process is analogous to the derivation of Eqs. (2.7) for the quasi-1D problem. In particular, the solution yields,

$$
\begin{aligned}
\frac{\partial u}{\partial t} & =\frac{\rho\left(a^{2}-u^{2}\right)\left(\frac{\partial M}{\partial t}\right)_{\beta} \frac{d u}{d M}+a_{0} M\left[\left(\frac{\partial M}{\partial t}\right)_{\beta}\left(\rho u \frac{d u}{d M}+\frac{d p}{d M}\right)+\kappa \rho a^{2} u\right]}{\rho\left[a^{2}-\left(u-a_{0} M\right)^{2}\right]}, \\
\frac{\partial p}{\partial t} & =\frac{u\left(a_{0} M-u\right)\left(\frac{\partial M}{\partial t}\right)_{\beta} \frac{d p}{d M}+a^{2}\left[\left(\frac{\partial M}{\partial t}\right)_{\beta}\left(\frac{d p}{d M}+a_{0} \rho M \frac{d u}{d M}\right)+a_{0} \kappa \rho u M\left(a_{0} M-u\right)\right]}{a^{2}-\left(u-a_{0} M\right)^{2}} .
\end{aligned}
$$

\subsection{Geometrical equivalence}

Using (2.20) to expand the expression $\left(p_{t}+\rho a u_{t}\right)$ then gives at any $\beta$ and $t$,

$$
\left(\frac{\partial M}{\partial t}\right)_{\beta}=-\frac{\frac{\kappa a_{0} M \rho a^{2} u}{(a+u)}+\left(\frac{a_{0} M}{a+u}-1\right)\left(\frac{\partial p}{\partial t}+\rho a \frac{\partial u}{\partial t}\right)}{\frac{d p}{d M}+\rho a \frac{d u}{d M}} .
$$

An expedited route to arrive at $(2.21)$ is to observe that because $v=0$, the momentum equation $(2.11 c)$ for $v$ decouples from the rest of Eqs. (2.11). Also comparing (2.11) to (2.1), and (2.4) to (2.19), the only difference is that $A^{\prime} / A$ in (2.1) is replaced by $\kappa$ in (2.11). As a result, (2.21) is directly analogous to (2.8). 
We can conclude that GSD in its complete form given by $(2.8)$ with the $\left(p_{t}+\rho a u_{t}\right)$ term retained, is equivalent to $2 \mathrm{D}$ Euler if and only if

$$
\kappa=A^{\prime} / A=\frac{1}{a_{0} M A}\left(\frac{\partial A}{\partial t}\right)_{\beta} .
$$

By defining $A$ in 2D flow as the normalized ray-tube area given in (2.10), Whitham (2011) (Eq. (8.59)) showed, using the standard $\alpha$ - $\beta$ curvilinear coordinates in GSD, that

$$
\frac{\partial \theta}{\partial \beta}=\frac{1}{M} \frac{\partial A}{\partial \alpha}
$$

where $\alpha \equiv a_{0} t$ is defined as a time-like label for the position of a shock along a ray corresponding to constant $\beta$. Equation (2.23) can be shown equivalent to (2.22) by noting $d s=A d \beta$. For example, the geometrical equivalence for a converging/diverging cylindrical shock of radius $r$ is trivially verified by identifying $A=A(r)=2 \pi r$, and thus $A^{\prime} / A=1 / r=\kappa$.

Notably, the geometrical equivalence between the evolution of shocks in quasi-1D and genuine 2D flows is invariant with respect to the Cartesian coordinates used in Sec. 2.2. Best (1993) observed this equivalence and derived an alternative to the original GGSD theory (Best 1991) resulting from defining the shock velocity to include the local flow velocity component that is tangential to the shock at a time instant. In contrast, in Sec. 2.2, we spatially parameterize the shock using $\beta$ that labels shock points that propagate locally normal to the shock front. This difference illustrates the somewhat arbitrary definition of the shock velocity. The formulations are physically equivalent. An immediate implication of the geometrical equivalence is that it suffices to consider the quasi-1D formulation alone in order to construct the shock dynamics in 2D, by effectively stacking ray tubes of normalized area $A$. We can thus proceed in the following analysis without invoking the $2 \mathrm{D}$ Euler equations.

\subsection{A hierarchical expansion of the Euler equations}

As shown in Sections. 2.1.1 and 2.3, compared to the true Euler equations, the nonuniform details of the flow behind the shock that is missing in the GSD approximation is entirely encapsulated in the quantity $\left(p_{t}+\rho a u_{t}\right)$. Therefore a suitable evolutionary model for such quantity is desired to provide corrections to the GSD description. In the present study, we adapt the GGSD model proposed by Best (1991), which expands the Euler equations as a hierarchical system that can be closed at any order. A summary of Best's formulation is outlined here.

The construction begins with assuming sufficient smoothness of quasi-1D flow and defining

$$
Q_{n} \equiv \frac{\partial^{n-1}}{\partial t^{n-1}}\left(\frac{\partial p}{\partial t}+\rho a \frac{\partial u}{\partial t}\right), \quad n=1,2, \ldots,
$$

evaluated immediately downstream the shock. It is noted that $Q_{1}$ gives the nonuniformity measure, the quantity of interest. Computing the expansion of $Q_{n}$ involves the following convenient differential operator,

$$
\mathcal{F}^{i, j} \equiv \frac{\partial^{i+j}}{\partial x^{i} \partial t^{j}}, \quad i, j \in\{0,1,2, \ldots\},
$$

acting on the flow variables $p, u, \rho$ and $a$. Let $\xi \in\{p, u, \rho\}$ : then along the trajectory of the shock, the total derivative of $\xi$ is given by

$$
\frac{D}{D t}\left(\mathcal{F}^{i, j}[\xi]\right)=\mathcal{F}^{i, j+1}[\xi]+a_{0} M \mathcal{F}^{i+1, j}[\xi]
$$


Further, the following identity for continuously differentiable functions $A$ and $B$,

$$
\mathcal{F}^{i, j}[A B]=\sum_{n=0}^{i} \sum_{m=0}^{j}\left(\begin{array}{c}
i \\
n
\end{array}\right)\left(\begin{array}{c}
j \\
m
\end{array}\right) \mathcal{F}^{n, m}[A] \mathcal{F}^{i-n, j-m}[B],
$$

where $\left(\begin{array}{l}i \\ n\end{array}\right)$ and $\left(\begin{array}{c}j \\ m\end{array}\right)$ are the binomial operators, allows the Euler equations (2.2) to be differentiated by the operator $\mathcal{F}^{i, j}$. This, together with (2.26), forms six linear equations for six unknowns:

$$
\left(\mathcal{F}^{i+1, j}[p], \mathcal{F}^{i+1, j}[u], \mathcal{F}^{i+1, j}[\rho], \mathcal{F}^{i, j+1}[p], \mathcal{F}^{i, j+1}[u], \mathcal{F}^{i, j+1}[\rho]\right)^{\mathrm{T}},
$$

which can be solved analogously to the derivation of (2.7). The explicit solutions are found in Eqs. (61)-(66) of Best (1991). Best's result can be viewed as extensions of the $\mathrm{RH}$ condition to the jump in partial derivatives of flow variables across a curved shock, which is a topic that has also been studied by Thomas (1947); Kanwal (1957); Pant (1969); Hornung (2010); Mölder (2016); Emanuel (2016, 2019).

With (2.28) determined, it is then possible to prove by induction the following dependency relation for the partial derivatives,

$$
\begin{aligned}
& \mathcal{F}^{0,0}[\xi]=\xi(M), \\
& \mathcal{F}^{i, j}[\xi]=\mathcal{F}^{i, j}[\xi]\left(M, Q_{1}, \ldots, Q_{k}, A^{\prime} / A, \ldots, \frac{d^{k-1}\left(A^{\prime} / A\right)}{d x^{k-1}}\right),
\end{aligned}
$$

for $k=i+j$, and $i, j \in\{0,1, \ldots\}$. With some manipulations, (2.29) leads to

$$
\begin{aligned}
& \frac{D M}{D t}=\frac{D M}{D t}\left(M, Q_{1}, A^{\prime} / A\right), \\
& \frac{D Q_{k+1}}{D t}=\frac{D Q_{k+1}}{D t}\left(M, Q_{1}, \ldots, Q_{k+2}, A^{\prime} / A, \ldots, \frac{d^{k}\left(A^{\prime} / A\right)}{d x^{k}}\right) .
\end{aligned}
$$

The explicit expansion of $(2.30 a)$ was earlier derived in (2.8) where only first-order partial derivatives of the flow variables were used. In general, higher order partial derivatives are required to expand $(2.30 b)$ and give

$$
\begin{aligned}
& \frac{D Q_{k+1}}{D t}=-\left[a_{0} M \frac{\partial^{k+1}}{\partial t^{k+1}}\left(\frac{\rho a^{2} u}{a+u}\right) A^{\prime} / A+a_{0} M \sum_{i=1}^{k+1}\left(\begin{array}{c}
k+1 \\
i
\end{array}\right) \frac{\partial^{i}}{\partial t^{i}}\left(\frac{1}{a+u}\right) Q_{k-i+2}\right. \\
& \left.+a_{0} M \frac{\partial^{k}}{\partial t^{k}}\left(\frac{\partial(\rho a)}{\partial t} \frac{\partial u}{\partial x}-\frac{\partial(\rho a)}{\partial x} \frac{\partial u}{\partial t}\right)+\left(\frac{a_{0} M}{a+u}-1\right) Q_{k+2}\right] .
\end{aligned}
$$

Importantly, $(2.29 b)$ and (2.30b) show that the dependence of $D Q_{k+1} / D t$ upon derivatives of $\xi$ of order $\leqslant k+1$ is fully specified by knowing $M$ and $Q_{i}$ of order $i \leqslant k+1$. Therefore, for a sequence of coupled nonlinear evolution equations described by $(2.30)$ for $k=1, \ldots, N$, closure can be achieved by truncating the term $\left[a_{0} M /(a+u)-1\right] Q_{N+2}$ in (2.31). This results in a closed system of $N+2$ nonlinear differential equations for the variables $M, Q_{1}, \ldots, Q_{N+1}$, that can be integrated in time provided the initial conditions for $M, Q_{1}, \ldots, Q_{N+1}$ are known.

For instance, it has been shown in Sec. 2.1.1 that truncation at $Q_{1}$ in $(2.30 a)$ leads to the $A$ - $M$ relation in GSD. And by sampling $Q_{1}$ and its higher derivatives $Q_{i \geqslant 1}$, at time $t=0$, the non-uniformity of the initial flow behind the shock is described, which then evolves in time according to the expansion of the Euler equations. Similar to applying the "characteristic rule" in GSD, where the $C^{+}$characteristic is applied at the shock, 
the truncation error associated with the present higher order approach is due to forcing the $D Q_{N} / D t$ equation along the $C^{+}$characteristic at the shock.

The conditions for convergence of the Mach number $M$ to the solution obtained from the full 2D Euler equations as a function of $t$, when the truncation number $N$ increases remains an open problem that is addressed incompletely in Best $(1991 ; 1993)$. We hypothesize that conditions for convergence may be problem dependent. It is noted that Best's formulation certainly breaks down when the smooth flow assumption is violated, especially if additional discontinuous disturbances that originated downstream and carried by the $C^{+}$characteristics overtake the shock. Such flow conditions are excluded in the present study.

\section{Evolution of a periodic, perturbed planar shock}

\subsection{A complex-variable formulation}

With the truncated GGSD equations established in Sec. 2.4, we can now describe the motion of a slightly perturbed planer shock, parameterized periodically using ray tube marker $\beta \in[0,2 \pi)$ [see Sec. 2.2], by correctly replacing $A(x)$ in the quasi-1D case with the normalized ray tube area given in (2.10) for a $2 \mathrm{D}$ shock. The $2 \pi$-periodic formulation is convenient for our application to a shock generated by a periodically corrugated piston in Section 4. Following Mostert et al. (2018a), we describe the shock shape in a complex $z=x+i y$ plane by $z=Z(\beta, t)=X(\beta, t)+i Y(\beta, t)$.

By defining ray tube markers along the shock whose velocity is locally normal to the shock curve, the kinematic equation for the shock profile reads as

$$
\left(\frac{\partial Z}{\partial t}\right)_{\beta}=a_{0} M(\beta, t) \hat{n}(\beta, t),
$$

where $Z \in \mathbb{C}$ is the complex variable that describes the shock curve, $a_{0}$ is the upstream constant sound speed, $M$ is the local Mach number and $\hat{n} \in \mathbb{C}$ is the unit normal vector aligned with the local shock velocity.

The complex shock profile $Z$ is now decomposed into a steady flat shock of constant Mach number $M_{0}$ moving in the positive imaginary direction, and its perturbation,

$$
Z=Z_{0}+z(\beta, t), \quad Z_{0}=\beta+i a_{0} M_{0} t .
$$

It is noted that the unperturbed shock at $t=0$ here lies on the real axis in the complex plane, and moves in the positive $y$-direction. This notation conventionally differs from the discussion in Sec. 2.2.

Further, the unit normal vector, $\hat{n}=i \hat{s}$, is obtained through a rotation of the unit tangent $\hat{s}$, given by

where

$$
\hat{s}=\frac{1}{A(\beta, t)} \frac{\partial Z}{\partial \beta},
$$

$$
A(\beta, t)=\left|\frac{\partial Z}{\partial \beta}\right|=\sqrt{\frac{\partial Z}{\partial \beta} \frac{\partial Z^{*}}{\partial \beta}},
$$

is the normalized ray tube area in the complex-variable formulation, following (2.10), and

* denotes complex conjugate. The kinematic equation for the shock profile perturbation then becomes,

$$
\frac{\partial z}{\partial t}=i a_{0} M_{0}\left[\frac{M}{M_{0}}\left(1+\frac{\partial z}{\partial \beta}\right)\left|1+\frac{\partial z}{\partial \beta}\right|^{-1}-1\right]
$$




\subsection{Zeroth-order GGSD system: GGSD-0}

In order to determine the evolution of the shock shape $z$, it remains to specify the dynamics of the Mach number profile $M$, which can also be written in the perturbation form

$$
M=M_{0}+m \text {. }
$$

Conveniently, using Eq. (2.21) with the help of (2.22) and (2.24), we have shown that the Euler equations dictates

$$
\frac{\partial m}{\partial t}=-\frac{\frac{\rho a^{2} u \Phi a_{0} M}{(a+u)}+\left(\frac{a_{0} M}{a+u}-1\right) Q_{1}}{d p / d M+\rho a(d u / d M)},
$$

where $\Phi \equiv(\partial A / \partial t) /\left(a_{0} M A\right)$ and can be obtained by differentiating (3.4) using (3.1) as

$$
\Phi=\Phi(Z)=-\left|\frac{\partial Z}{\partial \beta}\right|^{-3} \operatorname{Im}\left(\frac{\partial^{2} Z}{\partial \beta^{2}} \frac{\partial Z^{*}}{\partial \beta}\right) .
$$

Now, by setting $Q_{1}=0$, Eqs. (3.5) and (3.7) form a set of coupled non-linear differential equations that describes the shock motion. Such system corresponds to the zeroth-order truncation of the hierarchical GGSD equations introduced in Sec. 2.4, which gives rise to the $A-M$ relation seen in the GSD model.

There is a subtle difference between GSD and the zeroth-order GGSD system, henceforth referred to as GGSD-0. In GSD, the $A$ - $M$ relation, once determined with a unique integration constant, serves as a global constraint over the entire shock profile. For example, Mostert et al. (2018a) derived an expression for the Mach number profile $M$ for a planar shock that is completely determined by the local shock shape $Z$, namely, $M=M(Z)$. Consequently, Eqs. (3.5) and (3.7) reduce and combine into a single equation for $Z$ that governs the shock shape [see Eq. (2.9) of Mostert et al. (2018a)]. In contrast, by separating (3.5) and (3.7) as two independent evolution equations in GGSD-0, the $A-M$ relation applies locally at a given $\beta$, allowing the integration constant to differ along the shock. The obvious benefit of such separation is that GGSD-0 is able to handle more general initial value problems that are forbidden in GSD, where the initial shock shape and Mach number profile do not satisfy a global $A-M$ relation everywhere on the shock. Nevertheless if the initial conditions are compatible with a global $A-M$ relation, then GGSD-0 is identical to GSD.

\subsubsection{Strong and weak shock limits}

Here, we simplify Eq. (3.7) in the strong and weak shock limits, as $M \rightarrow \infty$ and $M \rightarrow 1$, respectively. This is achieved by utilising the limiting forms of the $\mathrm{RH}$ conditions (2.5) given by,

$$
u \sim \alpha_{1} a_{0} M, \quad p \sim \alpha_{1} \rho_{0} a_{0}^{2} M^{2}, \quad \rho \sim \alpha_{2} \rho_{0}, \quad a \sim \alpha_{3} a_{0} M
$$

as $M \rightarrow \infty$, where $\alpha_{1,2,3}$ are constant functions of $\gamma$, given in Table 1, Appendix A; and similarly,

$$
u \sim 2 \alpha_{1} a_{0} \varepsilon_{\mathrm{w}}, \quad p \sim \rho_{0} a_{0}^{2}\left(\frac{1}{\gamma}+2 \alpha_{1} \varepsilon_{\mathrm{w}}\right), \quad \rho \sim \rho_{0}\left(1+2 \alpha_{1} \varepsilon_{\mathrm{w}}\right), \quad a \sim a_{0}\left(1+\frac{2 \varepsilon_{\mathrm{w}}}{\alpha_{2}}\right),
$$

where $\varepsilon_{\mathrm{w}} \equiv M-1$ and $\varepsilon_{\mathrm{w}} \rightarrow 0$ defines the weak-shock limit. We note that Appendix A contains lists of parameters that we use throughout the present study. 
Evaluating (3.7) using Eqs. (3.9) and (3.10), respectively, then yields

$$
\left(\frac{\partial M}{\partial t}\right)_{\beta}= \begin{cases}-\zeta_{1} a_{0} \Phi M^{2}+\frac{\zeta_{2} Q_{1}}{\rho_{0} a_{0}^{2} M}+O(M), & M \rightarrow \infty \\ -\frac{a_{0} \Phi \varepsilon_{\mathrm{w}}}{2}+\frac{Q_{1} \varepsilon_{\mathrm{w}}}{4 \alpha_{1} \rho_{0} a_{0}^{2}}+O\left(\varepsilon_{\mathrm{w}}^{2}\right), & \varepsilon_{\mathrm{w}} \rightarrow 0\end{cases}
$$

where $\zeta_{1,2}$ are again constants listed in Table 1, Appendix A. In this asymptotic form, it is assumed that $Q_{1}$ is at least of order $O\left(M^{3}\right)$ in the strong shock limit, and of order $O\left(\varepsilon_{\mathrm{w}}^{0}\right)$ in the weak shock limit, for the correction term to be comparable in the respective leading order expression. Although not necessary for the zeroth-order GGSD model, where the correction is neglected by letting $Q_{1}=0$, such assumptions about the magnitude of $Q_{1}$ will be shown essential for deriving the limiting expressions for higher order GGSD models in the following sections.

For completeness, the zeroth-order GGSD systems in the strong and weak shock limits are given by the following closed system,

$$
\frac{\partial Z}{\partial t}=i a_{0} M \frac{\partial Z}{\partial \beta}\left|\frac{\partial Z}{\partial \beta}\right|^{-1}, \quad\left(\frac{\partial M}{\partial t}\right)_{\beta}= \begin{cases}-\zeta_{1} a_{0} \Phi M^{2}, & M \rightarrow \infty \\ -\frac{a_{0} \Phi}{2}(M-1), & M \rightarrow 1 .\end{cases}
$$

\subsection{First order GGSD system: GGSD-1}

As discussed in Sec. 2.4, corrections to GGSD-0 can be made by including higher order equations that capture the evolution of $Q_{1}$. In this section, we obtain the GGSD-1 model explicitly in the weak and strong shock limits.

The evolution of $Q_{1}$ following the shock trajectory in the Quasi-1D flow is governed by Eq. (2.31) with $k=0$. Using the present complex-variable formulation, the total time derivative, $D Q_{1} / D t$, described by Eq. (2.31) directly equates to the partial time derivative tracing a fixed ray tube marker $\beta$, namely, $\left(\partial Q_{1} / \partial t\right)_{\beta}$. Similarly, the full differential $d x$ that appears in $A^{\prime}$ is now understood as the differential distance traveled by the shock in the ray tube direction, i.e.,

$$
d x=a_{0} M(\beta, t) d t, \quad \frac{A^{\prime}}{A}=\frac{1}{a_{0} M A}\left(\frac{\partial A}{\partial t}\right)_{\beta}=\Phi,
$$

where $\Phi$ is given in (3.8). Now substituting (3.13) into (2.31) yields

$$
\begin{aligned}
\left(\frac{\partial Q_{1}}{\partial t}\right)_{\beta}=-a_{0} M\left[\frac{\partial}{\partial t}\left(\frac{\rho a^{2} u}{a+u}\right) \Phi\right. & +\frac{\partial(\rho a)}{\partial t} \frac{\partial u}{\partial x}-\frac{\partial(\rho a)}{\partial x} \frac{\partial u}{\partial t} \\
& \left.+\frac{\partial}{\partial t}\left(\frac{1}{a+u}\right) Q_{1}+\left(\frac{1}{a+u}-\frac{1}{a_{0} M}\right) Q_{2}\right] .
\end{aligned}
$$

Expanding (3.14) and noting $a=\sqrt{\gamma p / \rho}$ produces first order partial derivatives of $\rho$, $u$ and $p$ with respect to $t$ and $x$, which are found as a subset of solution (2.28), where $i=j=0$. Therefore in view of (2.29b), Eq.(3.14) is fully specified knowing $M, Q_{1}$ and $\Phi$, except for the last term that contains $Q_{2}$. Hence truncation at $Q_{2}=0$ provides closure. GGSD-1 then comprises (3.5), (3.7) and (3.14) with $Q_{2}=0$.

\subsubsection{The weak shock limit}

The full expansion of $\mathcal{F}^{0,1}\left[\xi^{*}\right]$ and $\mathcal{F}^{1,0}\left[\xi^{*}\right]$, where $\xi^{*} \in\{\rho, u, p, a\}$, can be obtained as a subset from the solution process to the arrival of (2.28). Using the asymptotic forms (3.10) and (3.11), these first order partial derivatives, listed in Eqs. (B 1)-(B 4) of Appendix $\mathrm{B}$, simplify in the weak shock limit as $\varepsilon_{\mathrm{w}}=M-1 \rightarrow 0$. It is noted that the leading 
order behaviour of all eight partial derivatives in this limit relies on the assumption that $Q_{1} \sim O\left(\varepsilon_{\mathrm{w}}^{0}\right) \sim O(1)$, which is consistent with the observation made with Eq. (3.11) when $Q_{1} \neq 0$ is allowed.

Substituting Eqs. (3.10) and (B 1)-(B 4) into (3.14) then gives the leading order evolution of $Q_{1}$ in the weak shock limit,

$$
\left(\frac{\partial Q_{1}}{\partial t}\right)_{\beta}=\frac{Q_{1}}{2}\left(\frac{Q_{1}}{\alpha_{1} \rho_{0} a_{0}^{2}}-a_{0} \Phi\right)+Q_{2} \varepsilon_{\mathrm{w}}+O\left(\varepsilon_{\mathrm{w}}\right)
$$

where the correction term $Q_{2} \varepsilon_{\mathrm{w}}$ enters only if $Q_{2} \sim O\left(1 / \varepsilon_{\mathrm{w}}\right)$ at least. However, closure of the GGSD system at first order demands $Q_{2}=0$; and therefore combining Eqs. (3.5), (3.11) and (3.15) completes the weak shock limiting GGSD-1 equations as follows,

$$
\begin{aligned}
& \frac{\partial Z}{\partial t}=i a_{0} M \frac{\partial Z}{\partial \beta}\left|\frac{\partial Z}{\partial \beta}\right|^{-1} \\
& \frac{\partial M}{\partial t}=\left(-\frac{a_{0} \Phi}{2}+\frac{Q_{1}}{4 \alpha_{1} \rho_{0} a_{0}^{2}}\right)(M-1) \\
& \frac{\partial Q_{1}}{\partial t}=\frac{Q_{1}}{2}\left(\frac{Q_{1}}{\alpha_{1} \rho_{0} a_{0}^{2}}-a_{0} \Phi\right) .
\end{aligned}
$$

\subsubsection{The strong shock limit}

Analogous analysis can be carried out for the strong shock limit. Substituting (3.9) into the general solution of the first order partial derivatives leads to the strong shock limits of Eqs. (B 1)-(B 4) in Appendix B , where $M^{\prime}$ is understood similarly as in (3.13), such that upon using (3.11), one has

$$
M^{\prime}=\frac{d M}{d x}=\frac{1}{a_{0} M}\left(\frac{\partial M}{\partial t}\right)_{\beta} \sim-\zeta_{1} \Phi M+\frac{\zeta_{2} Q_{1}}{\rho_{0} a_{0}^{3} M^{2}},
$$

It is thus observed that $M^{\prime} \sim O(M)$, so the $M^{\prime}$ notation is preferably kept in (B 1)-(B 4) and henceforth for convenient identification of the leading order behaviour.

Again, substituting (3.9), (B 1)-(B 4) and (3.17) into (3.14) yields the limiting $Q_{1}$ equation,

$$
\left(\frac{\partial Q_{1}}{\partial t}\right)_{\beta}=\gamma_{1} \rho_{0} a_{0}^{4} \Phi^{2} M^{4}+\gamma_{2} a_{0} M \Phi Q_{1}+\frac{\gamma_{3} Q_{1}^{2}}{\rho_{0} a_{0}^{2} M^{2}}+\gamma_{4} Q_{2}+O\left(M^{3}\right)
$$

where $\gamma_{1,2,3,4}$ are coefficients listed in Table 1 , Appendix A. Setting $Q_{2}=0$, which otherwise is assumed as $Q_{2} \sim O\left(M^{4}\right)$, Eq. (3.18) is joined with (3.5) and (3.11) to form the GGSD-1 equations in the strong shock limit,

$$
\begin{aligned}
& \frac{\partial Z}{\partial t}=i a_{0} M \frac{\partial Z}{\partial \beta}\left|\frac{\partial Z}{\partial \beta}\right|^{-1}, \\
& \frac{\partial M}{\partial t}=-\zeta_{1} a_{0} \Phi M^{2}+\frac{\zeta_{2} Q_{1}}{\rho_{0} a_{0}^{2} M} \\
& \frac{\partial Q_{1}}{\partial t}=\gamma_{1} \rho_{0} a_{0}^{4} \Phi^{2} M^{4}+\gamma_{2} a_{0} \Phi M Q_{1}+\frac{\gamma_{3} Q_{1}^{2}}{\rho_{0} a_{0}^{2} M^{2}} .
\end{aligned}
$$




\subsection{Second order GGSD system: GGSD-2}

The second order GGSD system can be similarly established by incorporating the evolution of $Q_{2}$, governed by Eq. (2.31) where $k=1$ and $Q_{3}=0$,

$$
\begin{array}{r}
\left(\frac{\partial Q_{2}}{\partial t}\right)_{\beta}=-a_{0} M \Phi \frac{\partial^{2}}{\partial t^{2}}\left(\frac{\rho a^{2} u}{a+u}\right)-a_{0} M\left[\frac{\partial^{2}}{\partial t^{2}}\left(\frac{1}{a+u}\right) Q_{1}+2 \frac{\partial}{\partial t}\left(\frac{1}{a+u}\right) Q_{2}\right] \\
-a_{0} M \frac{\partial}{\partial t}\left(\frac{\partial(\rho a)}{\partial t} \frac{\partial u}{\partial x}-\frac{\partial(\rho a)}{\partial x} \frac{\partial u}{\partial t}\right)-\left(\frac{a_{0} M}{a+u}-1\right) Q_{3} .
\end{array}
$$

Again, without the $Q_{3}$ correction, the rest of (3.20) is fully determined because the second order partial derivatives, $\left\{\mathcal{F}^{n, m}\left[\xi^{*}\right]: \xi^{*} \in\{\rho, u, p, a\}, n+m=2\right\}$, only depends on $M$, $Q_{1}, Q_{2}$ and ultimately $Z$. To see this, the apparent dependency of $\left(A^{\prime} / A\right)^{\prime}$ in $(2.29 b)$ for quasi-1D flow here becomes

$$
\frac{d}{d x}\left(\frac{A^{\prime}}{A}\right)=\frac{1}{a_{0} M}\left(\frac{\partial \Phi}{\partial t}\right)_{\beta}
$$

which can be evaluated according to the definition of $\Phi(3.8)$, after obtaining the partial derivatives, $Z_{\beta t}$ and $Z_{\beta \beta t}$, from differentiating (3.1) and its complex conjugate with respect to $\beta$, once and twice, respectively. As a result, one defines $\Psi \equiv\left(A^{\prime} / A\right)^{\prime}$ and arrives at

$$
\Psi=\Psi(Z, M)=-\Phi^{2}-\frac{1}{A^{4} M}\left[A^{2} \frac{\partial^{2} M}{\partial \beta^{2}}-\operatorname{Re}\left(\frac{\partial^{2} Z}{\partial \beta^{2}} \frac{\partial Z^{*}}{\partial \beta}\right) \frac{\partial M}{\partial \beta}\right]
$$

where $A=|\partial Z / \partial \beta|$. The explicit expansion of (3.20) is determined next in the weak and strong shock limits.

\subsubsection{The weak shock limit}

In order to obtain the various second order partial derivatives involved in (3.20), the shock local acceleration is also required. This is computed by differentiating (3.11) (recall $\epsilon=M-1)$. as follows,

$$
\frac{\partial^{2} \varepsilon_{\mathrm{w}}}{\partial t^{2}}=\frac{1}{4 \alpha_{1} \rho_{0}^{2} a_{0}^{2}}\left\{\varepsilon_{\mathrm{w}} \frac{\partial Q_{1}}{\partial t}+Q_{1} \frac{\partial \varepsilon_{\mathrm{w}}}{\partial t}-2 \alpha_{1} \rho_{0}^{2} a_{0}^{3}\left[\Phi \frac{\partial \varepsilon_{\mathrm{w}}}{\partial t}+a_{0} \varepsilon_{\mathrm{w}}\left(1+\varepsilon_{\mathrm{w}}\right) \Psi\right]\right\} \sim O\left(\varepsilon_{\mathrm{w}}\right),
$$

where $\partial Q_{1} / \partial t, \partial \varepsilon_{\mathrm{w}} / \partial t, \Phi$ and $\Psi$ are known due to Eqs. (3.15), (3.11), (3.8) and (3.22).

With (3.10), (B 1)-(B 4), (3.22) and (3.23) available, expressions for the required second- order partials of flow variables are Taylor expanded around $\varepsilon_{\mathrm{w}}=0$, yielding the leading order behaviour given in Eqs. (B 5)-(B 8) of Appendix B. We note that $Q_{2} \sim O\left(1 / \varepsilon_{\mathrm{w}}\right)$ and recall (3.15). Finally, substituting (3.10) and (B 5)-(B 8) into (3.20) while letting $Q_{3}=0$ produces the evolution equation for $Q_{2}$ as $\epsilon \rightarrow 0$, which is then joined to (3.5), (3.11) and (3.15) to form the following GGSD-2 system in the weak 
shock limit,

$$
\begin{aligned}
\frac{\partial Z}{\partial t} & =i a_{0} M \frac{\partial Z}{\partial \beta}\left|\frac{\partial Z}{\partial \beta}\right|^{-1}, \\
\frac{\partial M}{\partial t} & =(M-1)\left(-\frac{a_{0} \Phi}{2}+\frac{Q_{1}}{4 \alpha_{1} \rho_{0} a_{0}^{2}}\right), \\
\frac{\partial Q_{1}}{\partial t} & =\frac{Q_{1}}{2}\left(\frac{Q_{1}}{\alpha_{1} \rho_{0} a_{0}^{2}}-a_{0} \Phi\right)+(M-1) Q_{2}, \\
\frac{\partial Q_{2}}{\partial t} & =\frac{\left[(\gamma+1) Q_{1}-2 \rho_{0} a_{0}^{3} \Phi\right] Q_{2}}{4 \rho_{0} a_{0}^{2}} .
\end{aligned}
$$

\subsubsection{The strong shock limit}

As an analogy to (3.23), the second time derivative $\partial^{2} M / \partial t^{2}$ is prepared first

$$
\frac{\partial^{2} M}{\partial t^{2}}=-\zeta_{1} a_{0} M\left(a_{0} \Psi M^{2}+2 \Phi \frac{\partial M}{\partial t}\right)+\frac{\zeta_{2}}{\rho_{0} a_{0}^{2} M^{2}}\left(M \frac{\partial Q_{1}}{\partial t}-Q_{1} \frac{\partial M}{\partial t}\right),
$$

where $\partial Q_{1} / \partial t, \partial M / \partial t, \Phi$ and $\Psi$ are found in (3.18), (3.11), (3.8) and (3.22), respectively. However here in the strong shock limit, it is more convenient to further attain $M^{\prime \prime} \equiv$ $d\left(M^{\prime}\right) / d x$, fully expanded using (3.13), (3.11), (3.18) and (3.25) as

$$
M^{\prime \prime}=\left[\left(\zeta_{1}^{2}+\gamma_{1} \zeta_{2}\right) \Phi^{2}-\zeta_{1} \Psi\right] M+\frac{\left(\zeta_{1}+\gamma_{2}\right) \zeta_{2} \Phi Q_{1}}{\rho_{0} a_{0}^{3} M^{2}}+\frac{\zeta_{2}\left(\gamma_{3}-2 \zeta_{2}\right) Q_{1}^{2}}{\rho_{0}^{2} a_{0}^{6} M^{5}}+\frac{\zeta_{2} \gamma_{4} Q_{2}}{\rho_{0} a_{0}^{4} M^{3}} .
$$

Consequently, $M^{\prime \prime} \sim M^{\prime} \sim O(M)$ is established.

Next, the second order partial derivatives contributing to the expansion of $\partial Q_{2} / \partial t$ can be expressed in terms of $M^{\prime \prime}$, for the same reason that $M^{\prime}$ is preferred in Eqs. (B 1)(B 4). The explicit asymptotic formulas are reported in Eqs. (B 5)-(B 8), Appendix B. After evaluating (3.20) using Eqs. (B 5)-(B 8) in the strong shock limit, the GGSD-2 equations follows,

$$
\begin{aligned}
\frac{\partial Z}{\partial t} & =i a_{0} M \frac{\partial Z}{\partial \beta}\left|\frac{\partial Z}{\partial \beta}\right|^{-1}, \\
\frac{\partial M}{\partial t} & =-\zeta_{1} a_{0} \Phi M^{2}+\frac{\zeta_{2} Q_{1}}{\rho_{0} a_{0}^{2} M}, \\
\frac{\partial Q_{1}}{\partial t} & =\gamma_{1} \rho_{0} a_{0}^{4} \Phi^{2} M^{4}+\gamma_{2} a_{0} \Phi M Q_{1}+\frac{\gamma_{3} Q_{1}^{2}}{\rho_{0} a_{0}^{2} M^{2}}+\gamma_{4} Q_{2}, \\
\frac{\partial Q_{2}}{\partial t} & =\rho_{0} a_{0}^{5} M^{5}\left(\chi_{1} \Phi \Psi+\chi_{2} \Phi^{3}\right)-a_{0}^{2} M^{2}\left(\chi_{3} \Psi+\chi_{4} \Phi^{2}\right) Q_{1}-\frac{\chi_{5} \Phi Q_{1}^{2}}{\rho_{0} a_{0} M} \\
& \quad+\frac{\chi_{6} Q_{1}^{3}}{\rho_{0}^{2} a_{0}^{4} M^{4}}+\frac{\chi_{7} Q_{1} Q_{2}}{\rho_{0} a_{0}^{2} M^{2}}+\chi_{8} a_{0} \Phi M Q_{2},
\end{aligned}
$$

where $\chi_{1,2, \ldots, 8}$ are again constant functions of $\gamma$ listed in Table 1, Appendix A. This completes the derivation of shock-evolution equations used presently.

\subsection{Unbounded linear growth}

We now demonstrate that the zeroth-order GGSD system is linearly stable, whilst including the $Q_{1}$ correction in the first order GGSD equations leads to linearised solutions that exhibit unbounded growth in time. Equations (3.16) and (3.19) are expanded around a plane shock of constant Mach number $M_{0}$, and consequently a steady downstream flow 
with $Q_{1}=Q_{10}$. The plane shock motion is described by $Z_{0}=\beta+i a_{0} M_{0} t$, and the following rescaled perturbation fields are considered,

$$
Z=Z_{0}+z_{1}(\beta, t) \delta+\ldots, \quad M=M_{0}+m_{1}(\beta, t) \delta+\ldots, \quad Q_{1}=Q_{10}+q_{1}(\beta, t) \delta+\ldots,
$$

where $\delta \ll 1$ is the dimensionless perturbation size, and the subscript " 1 " is used to distinguish from unscaled variables used in (3.2) and (3.6). The $\delta \rightarrow 0$ asymptotic behaviour is explored next for the limiting equations (3.16) and (3.19), corresponding to weak and strong shocks where the respective $M_{0} \rightarrow 1$ and $M_{0} \rightarrow \infty$ limits have been applied. Substituting (3.28) into Eqs. (3.16) and (3.19) yields, at order $O\left(\delta^{0}\right)$, that for both weak and strong shocks,

$$
Q_{10}=0,
$$

implying that the linear behaviour of the shock is fully described by the zeroth-order GGSD system. Further, at order $O(\delta)$, one has

$$
\begin{aligned}
& \frac{\partial z_{1}}{\partial t}=\frac{i a_{0} M_{0}}{2}\left(\frac{\partial z}{\partial \beta}-\frac{\partial z^{*}}{\partial \beta}\right)+i a_{0} m_{1}, \\
& \frac{\partial m_{1}}{\partial t}= \begin{cases}\frac{\zeta_{2} q_{1}}{\rho_{0} a_{0}^{2} M_{0}}-\frac{i \zeta_{1} a_{0} M_{0}^{2}}{2}\left(\frac{\partial^{2} z_{1}}{\partial \beta^{2}}-\frac{\partial^{2} z_{1}^{*}}{\partial \beta^{2}}\right), & M_{0} \rightarrow \infty \\
\frac{\left(M_{0}-1\right) q_{1}}{4 \alpha_{1} \rho_{0} a_{0}^{2}}-\frac{i a_{0}\left(M_{0}-1\right)}{4}\left(\frac{\partial^{2} z_{1}}{\partial \beta^{2}}-\frac{\partial^{2} z_{1}^{*}}{\partial \beta^{2}}\right), & M_{0} \rightarrow 1,\end{cases} \\
& \frac{\partial q_{1}}{\partial t}=0
\end{aligned}
$$

As a result of $(3.30 c), q_{1}$ is a constant function of $\beta$ to be determined from the initial conditions of the flow immediately behind the shock, namely,

$$
q_{1}(\beta, t)=q_{1}(\beta, 0), \quad \forall t>0 .
$$

By differentiating $(3.30 a)$ with respect to $\beta,(3.30 b)$ can be cast into

$$
\frac{\partial m_{1}}{\partial t}= \begin{cases}-\frac{\zeta_{1} M_{0}}{2} \frac{\partial}{\partial t}\left(\frac{\partial z_{1}}{\partial \beta}+\frac{\partial z_{1}^{*}}{\partial \beta}\right)+\frac{\zeta_{2} q_{1}}{\rho_{0} a_{0}^{2} M_{0}}, & M_{0} \rightarrow \infty \\ -\frac{\left(M_{0}-1\right)}{4 M_{0}} \frac{\partial}{\partial t}\left(\frac{\partial z_{1}}{\partial \beta}+\frac{\partial z_{1}^{*}}{\partial \beta}\right)+\frac{\left(M_{0}-1\right) q_{1}}{4 \alpha_{1} \rho_{0} a_{0}^{2}}, & M_{0} \rightarrow 1,\end{cases}
$$

which can be integrated with respect to $t$ because of (3.31) to give

$$
m_{1}= \begin{cases}-\frac{\zeta_{1} M_{0}}{2}\left(\frac{\partial z_{1}}{\partial \beta}+\frac{\partial z_{1}^{*}}{\partial \beta}\right)+\frac{\zeta_{2} q_{1}}{\rho_{0} a_{0}^{2} M_{0}} t+f(\beta), & M_{0} \rightarrow \infty \\ -\frac{M_{0}-1}{4}\left(\frac{\partial z_{1}}{\partial \beta}+\frac{\partial z_{1}^{*}}{\partial \beta}\right)+\frac{\left(M_{0}-1\right) q_{1}}{4 \alpha \rho_{0} a_{0}^{2}} t+f(\beta), & M_{0} \rightarrow 1,\end{cases}
$$

where $f(\beta)$ is prescribed from the initial Mach number profile $m_{1}(\beta, 0)$. Further, (3.33) is substituted into $(3.30 a)$, to show

$$
\frac{\partial z_{1}}{\partial \tau}= \begin{cases}i\left(\frac{1-\zeta_{1}}{2} \frac{\partial z}{\partial \beta}-\frac{1+\zeta_{1}}{2} \frac{\partial z^{*}}{\partial \beta}+\frac{\zeta_{2} q_{1} \tau}{\rho_{0} a_{0}^{3} M_{0}^{3}}+\frac{f}{M_{0}}\right), & M_{0} \rightarrow \infty \\ i\left(\frac{3-M_{0}}{4} \frac{\partial z_{1}}{\partial \beta}-\frac{1+M_{0}}{4} \frac{\partial z_{1}^{*}}{\partial \beta}+\frac{\left(M_{0}-1\right) q_{1} \tau}{4 \alpha_{1} \rho_{0} a_{0}^{3}}+f\right), & M_{0} \rightarrow 1\end{cases}
$$

where $\tau=a_{0} M_{0} t$ is a time-like length unit defined for convenience. The solution for $z_{1}$ can be obtained using a Fourier expansion in $\beta$,

$$
z_{1}(\beta, \tau)=\sum_{n=-\infty}^{\infty} \hat{z}_{n}(\tau) e^{i n \beta}, \quad q_{1}(\beta)=\sum_{n=-\infty}^{\infty} \hat{q}_{n} e^{i n \beta}, \quad f(\beta)=\sum_{n=-\infty}^{\infty} \hat{f}_{n} e^{i n \beta},
$$


in both (3.34) and its complex conjugate. This yields the following second order ordinary differential equations for the Fourier coefficients $\hat{z}_{n}$,

$$
\frac{d^{2} \hat{z}_{n}}{d \tau^{2}}= \begin{cases}-\zeta_{1} n^{2} \hat{z}_{n}+i\left[\frac{\zeta_{2}(1-n \tau) \hat{q}_{n}}{\rho_{0} a_{0}^{3} M_{0}^{3}}-\frac{n \hat{f}_{n}}{M_{0}}\right], & M_{0} \rightarrow \infty \\ -\frac{M_{0}-1}{2} n^{2} \hat{z}_{n}+i\left[\frac{\left(M_{0}-1\right)(1-n \tau) \hat{q}_{n}}{4 \alpha_{1} \rho_{0} a_{0}^{3}}-n \hat{f}_{n}\right], & M_{0} \rightarrow 1\end{cases}
$$

whose solutions are given by

$$
\hat{z}_{n}= \begin{cases}A_{n} \cos \left(\sqrt{\zeta_{1}} n \tau\right)+B_{n} \sin \left(\sqrt{\zeta_{1}} n \tau\right)+i\left(C_{n} \tau+D_{n}\right), & M_{0} \rightarrow \infty, \\ A_{n} \cos \left(\sqrt{\frac{M_{0}-1}{2}} n \tau\right)+B_{n} \sin \left(\sqrt{\frac{M_{0}-1}{2}} n \tau\right)+i\left(C_{n} \tau+D_{n}\right), & M_{0} \rightarrow 1,\end{cases}
$$

where $A_{n}$ and $B_{n}$ are constants to be determined from the initial shock shape $z_{1}(\beta, 0)$; and from the particular linear source term,

$$
C_{n}=\left\{\begin{array}{ll}
\frac{-\zeta_{2} \hat{q}_{n}}{\zeta_{1} \rho_{0} a_{0}^{3} M_{0}^{3} n^{2}}, \\
\frac{-\hat{q}_{n}}{2 \alpha_{1} \rho_{0} a_{0}^{3} n},
\end{array} \quad D_{n}= \begin{cases}\frac{\zeta_{2} \hat{q}_{n}-\rho_{0} a_{0}^{3} M_{0}^{2} n \hat{f}_{n}}{\zeta_{1} \rho_{0} a_{0}^{3} M_{0}^{3} n^{2}}, & M_{0} \rightarrow \infty \\
\frac{1}{2 n^{2}}\left(\frac{\hat{q}_{n}}{\alpha_{1} \rho_{1} a_{0}^{3}}-\frac{4 n \hat{f}_{n}}{M_{0}-1}\right), & M_{0} \rightarrow 1\end{cases}\right.
$$

Finally substituting (3.38) into (3.35) and subsequently (3.33) completes the general solutions for $z_{1}$ and $m_{1}$.

If a shock is given an initial Mach number perturbation, and the flow that produced the perturbation persists, a reasonable physical expectation could be that the shock develops a shape perturbation that grows linearly in the imaginary (shock normal) direction with time. In the present theory, the forcing behind the shock is represented by the quantities $Q_{1}$ and $Q_{2}$. From Eqs. (3.38) and (3.33), for a flow with $Q_{1}=0$, where the shock is given an initial Mach number perturbation and moves into a uniform medium, linear theory produces a solution that is the same as would be predicted by the linear zeroth-order GGSD model (or GSD), where $Q_{1}$ plays no role. Here the shock motion consists of stable oscillations in the shock shape that are normal to its unperturbed profile, and with fixed amplitude and frequency.

For the present piston-generated shock, where the shock moves into a uniform medium but is subject to an initial perturbation with $Q_{1} \neq 0$, linearised first-order GGSD predicts, in addition to oscillatory behavior, a growth term that is linear in time for both the shock shape and Mach number perturbations, consistent with the above-stated expectation. Hence the linearised GGSD model suggests unbounded shock acceleration driven by nonuniformities (that is, nonzero $Q_{1}$ ) behind the shock. This amplitude growth, however, does not occur in alternative theories [e.g, Freeman (1957)]. In the sequel it will be shown that linear growth in time is in fact controlled by nonlinear behaviour.

\section{Evolution of shock generated by a slightly corrugated piston}

We now use the GGSD equations to investigate the evolution and nonlinear stability of a shock generated by an impulsively started piston of sinusoidal shape. The GGSD approach is chosen over numerical solutions of the full Euler equations. Shock-capturing Euler methods contain their own shock modeling in the form of highly nonlinear artificial dissipation focused on the shock profile, making them a questionable choice for investigating the long-time evolution of tiny disturbances to a plane shock. A shock-fitting method would seem ideal but is difficult to implement at order sufficiently high to accurately capture spontaneous singularity formation. 


\subsection{Base motion}

The shock considered in this study is produced by the impulsive motion of a piston from rest, whose velocity as a complex variable in the $Y$-direction is given by

$$
U_{p}(t)=U H(t) e^{i \pi / 2},
$$

where $U>0$ is the constant speed that the piston gains and $H(t)$ is the Heaviside function. For a piston with flat surface located at $Y=0$ moving into a uniform stationary medium, such impulse in the limit of $U \rightarrow \infty$ or $U \rightarrow 0$ generates a normal plane shock of constant Mach number,

$$
M_{0}=\left\{\begin{array}{l}
\frac{U}{\alpha_{1} a_{0}}, M \rightarrow \infty, \\
1+\frac{U}{2 a_{0} \alpha_{1}}, M \rightarrow 1,
\end{array}\right.
$$

after applying the strong and weak shock limits of the $\mathrm{RH}$ conditions [equations (3.9) and (3.10)]. The base motion of the shock in the form of (3.2) is thus established, namely,

$$
Z_{0}=\beta+i a_{0} M_{0} t
$$

It is noted that for $t>0$, the shock-processed flow between the piston and shock front is uniform with velocity also given by (4.1). To proceed, non-dimensional variables are used according to a reference length scale $L_{0}$, such that $2 \pi L_{0}$ is the initial perturbation wavelength; a reference velocity $a_{0}$ and density $\rho_{0}$. The time and pressure scales are thus derived as $L_{0} / a_{0}$ and $\rho_{0} a_{0}^{2}$, respectively.

\subsection{Initial values for $Z$ and $M$}

Next, a cosine perturbation of small amplitude $\epsilon \ll 1$ is imposed to the piston surface. Freeman (1955) showed that the corresponding shock generated by such corrugated piston must have the same initial shape profile as the piston surface. Therefore, at $t=0^{+}$, the shock shape is prescribed as

$$
Z\left(\beta, 0^{+}\right)=Z_{0}\left(\beta, 0^{+}\right)+z\left(\beta, 0^{+}\right)=\beta+i \epsilon \cos (\beta) .
$$

In order to obtain the initial Mach number distribution of this curved shock produced by the same piston impulse (4.1), it is noted that along the infinitely thin layer of fluid sandwiched between the piston and shock at $t=0^{+}$, only the piston velocity component locally normal to the boundary is transferred into the fluid, whereas the tangential component slips through the fluid under the present Euler description. Therefore in the laboratory frame where the piston velocity (4.1) is measured, the initial boundary fluid velocity distribution follows as the projection of piston velocity onto the unit normal vector given in (3.3), leading to

$$
u_{0} \equiv u\left(\beta, 0^{+}\right)= \begin{cases}\frac{\alpha_{1} M_{0}}{\sqrt{\epsilon^{2} \sin ^{2}(\beta)+1}}, & M_{0} \rightarrow \infty, \\ \frac{2 \alpha_{1}\left(M_{0}-1\right)}{\sqrt{\epsilon^{2} \sin ^{2}(\beta)+1}}, & M_{0} \rightarrow 1,\end{cases}
$$

where the base motion Mach number $M_{0}$ found in (4.2) is used as the input parameter. Because the shock curve coincides with the piston surface at $t=0^{+}$, the normal $\mathrm{RH}$ conditions can be applied again to convert (4.5) into the required initial values of Mach number,

$$
M\left(\beta, 0^{+}\right)= \begin{cases}\frac{M_{0}}{\sqrt{\epsilon^{2} \sin ^{2}(\beta)+1}}, & M_{0} \rightarrow \infty \\ 1+\frac{M_{0}-1}{\sqrt{\epsilon^{2} \sin ^{2}(\beta)+1}}, & M_{0} \rightarrow 1\end{cases}
$$


(a)

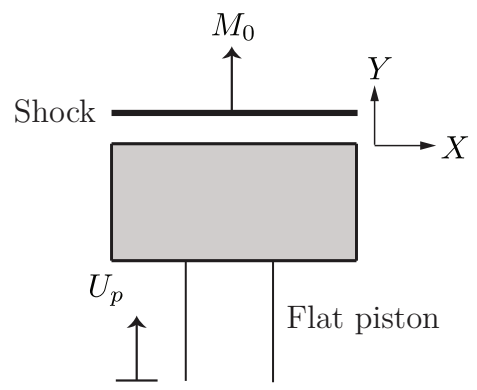

(b)

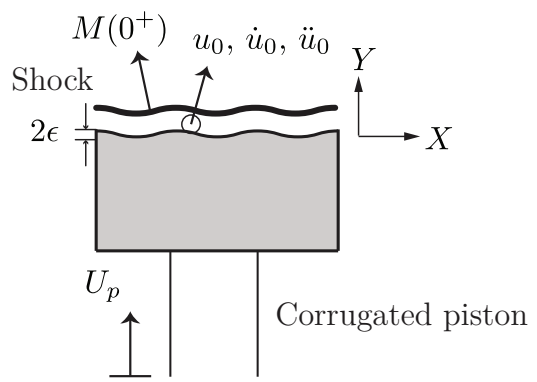

FIGURE 2. Shock generated by a flat piston shown in (a), and a corrugated piston of perturbation amplitude $\epsilon$ shown in (b), at time $t=0^{+}$. Both pistons share the same velocity $U_{p}$, giving a constant Mach number $M_{0}$ when the piston is flat. In (b) the Mach number profile is $M\left(\beta, 0^{+}\right)$. The flow between the piston and the shock is uniform in (a), but non-uniform with initial velocity $u_{0}$, acceleration $\dot{u}_{0}$, and jerk $\ddot{u}_{0}$ in (b).

A schematic comparison between shocks generated by flat and corrugated pistons is shown in figure 2, where the infinitesimal layer of shock-processed flow attached to the piston boundary at $t=0^{+}$is illustrated with kinematic quantities including acceleration and jerk that are discussed in the next section.

\subsection{Initial values for $Q_{1}$ and $Q_{2}$}

To complete the GGSD initial value problem formulation for the piston generated shock, it remains to determine $Q_{1}$ and $Q_{2}$ at $t=0^{+}$. This is achieved by utilising two kinematic conditions of the boundary flow, about its initial acceleration $\dot{u}_{0}$ and jerk $\ddot{u}_{0}$ in the shock normal direction. For each $\beta$, these quantities take the form of a full derivative moving with the piston,

$$
\begin{aligned}
& \dot{u}_{0}(\beta)=u_{t}\left(\beta, 0^{+}\right)+u_{0} u_{x}\left(\beta, 0^{+}\right), \\
& \ddot{u}_{0}(\beta)=\left.\frac{\partial\left(u_{t}+u u_{x}\right)}{\partial t}\right|_{t=0^{+}}+\left.u_{0} \frac{\partial\left(u_{t}+u u_{x}\right)}{\partial x}\right|_{t=0^{+}},
\end{aligned}
$$

where $u_{0}$ is derived in (4.5), and the first and second order derivatives of $u$ are given in Eqs. (B 1)-(B 4) and (B 5)-(B 8), respectively, in Appendix B.

Expanding (4.7a) using (B $2 a)$ and (B 2b), while in the strong shock case expressing $M^{\prime}$ in terms of $Q_{1}$ via (3.17) leads to the initial values for $Q_{1}$,

$$
Q_{1}\left(\beta, 0^{+}\right)= \begin{cases}\frac{M\left(\beta, 0^{+}\right)^{2}}{\zeta_{2}}\left[\zeta_{1} \Phi M+\frac{4 \gamma M\left(u_{0}-1\right) \Phi+(\gamma+1)^{2} \dot{u}_{0}}{2(\gamma+1)\left(4 M-3 u_{0}\right)}\right]_{t=0^{+}}, & M_{0} \rightarrow \infty, \\ \left.\frac{4(M-1) \Phi\left(-2 \gamma+(3 \gamma-1) u_{0}+2\right)+2(\gamma+1)^{2} \dot{u}_{0}}{(\gamma+1)^{2}\left(2 M-u_{0}-1\right)}\right|_{t=0^{+}}, & M_{0} \rightarrow 1,\end{cases}
$$

where in both limits, $\left.\Phi\right|_{t=0^{+}}$can be evaluated from its definition (3.8) using the initial shock profile (4.4) as

$$
\Phi\left(\beta, 0^{+}\right)=\frac{\epsilon \cos (\beta)}{\left(\epsilon^{2} \sin ^{2}(\beta)+1\right)^{3 / 2}} .
$$

Substituting (4.5), (4.6) and (4.9) into (4.8) completes the expression. Specifically, evaluating $Q_{1}\left(\beta, 0^{+}\right)$at $\epsilon=0$ shows

$$
\left.Q_{1}\left(\beta, 0^{+}\right)\right|_{\epsilon=0}= \begin{cases}\frac{(\gamma+1) M_{0} \dot{u}_{0}(\beta)}{\left(8-6 \alpha_{1}\right) \zeta_{2}}, & M_{0} \rightarrow \infty \\ \frac{2 \dot{u}_{0}(\beta)}{1-2\left(\alpha_{1}-1\right)\left(M_{0}-1\right)}, & M_{0} \rightarrow 1\end{cases}
$$

As discussed in Sec. 4.1, the unperturbed flow between the piston and shock is uniform, 
implying that $\left.Q_{1}\left(\beta, 0^{+}\right)\right|_{\epsilon=0}=0$ for all $\beta$. Therefore it is concluded that $\dot{u}_{0}(\beta)=0$, that is, the initial boundary flow is acceleration free. Hence $Q_{1}\left(\beta, 0^{+}\right)$simplifies to yield the following leading order behaviour as $\epsilon \rightarrow 0$,

$$
Q_{1}\left(\beta, 0^{+}\right) \sim \begin{cases}\frac{M_{0}^{3} \epsilon \cos (\beta)\left[\alpha_{1}\left(3(\gamma+1) \zeta_{1}-2 \gamma\right)-4(\gamma+1) \zeta_{1}\right]}{\left(3 \alpha_{1}-4\right)(\gamma+1) \zeta_{2}}, & M_{0} \rightarrow \infty, \\ \frac{8\left(M_{0}-1\right) \epsilon(1-\gamma) \cos (\beta)}{(\gamma+1)^{2}}, & M_{0} \rightarrow 1 .\end{cases}
$$

We note that our conclusion that the initial boundary flow along the piston surface has zero acceleration, with consequence (4.11), does not involve truncation error associated with the GGSD systems. Instead the presence of the $Q_{1}$ correction in the evolution equation of $M$ allows its initial condition to be inferred without knowledge of higher order corrections $Q_{i \geqslant 2}$.

Next, expanding $(4.7 b)$ in terms of $u_{t}, u_{x}, u_{t t}, u_{t x}$ and $u_{x x}$ evaluated at $t=0^{+}$ produces a linear equation from which the initial value of $Q_{2}$ can be solved. With a similar derivation provided in Appendix $\mathrm{C}$, it is shown again that the initial flow attached to the piston boundary has to be jerk free, i.e., $\ddot{u}_{0}=0$, in order to recover the unperturbed uniform result. And thus in Appendix C, the asymptotic form of $Q_{2}\left(\beta, 0^{+}\right)$for small $\epsilon$ follows,

$$
Q_{2}\left(\beta, 0^{+}\right) \sim \begin{cases}\left(\xi_{1} \cos ^{2}(\beta)+\xi_{2} \sin ^{2}(\beta)\right) M_{0}^{4} \epsilon^{2}, & M_{0} \rightarrow \infty, \\ -\frac{32 \gamma \cos ^{2}(\beta)\left(M_{0}-1\right)^{3} \epsilon^{2}}{(\gamma+1)^{3}}, & M_{0} \rightarrow 1,\end{cases}
$$

where $\xi_{1}$ and $\xi_{2}$ are coefficient functions of $\gamma$ tabulated in Table 2, Appendix A.

With initial conditions served by Eqs. (4.4), (4.6), (4.11) and (4.12), evolution of the shock generated by the perturbed piston can be tracked by the GGSD systems up to second order, by integrating in time the system of equations (3.24) and (3.27) for weak and strong shocks, respectively. While at $t=0$, the initial values for $M, Q_{1}$, and $Q_{2}$ can be obtained faithfully from the full 2D Euler equations, the GGSD construction does not directly propagate additional flow disturbances due to the piston motion, and carried by the $C^{+}$characteristics when $t>0$. Our adoption of the GGSD theory therefore assumes that perturbations caused by the corrugated piston after the initial impulse are small and smooth, while sufficiently far from the piston wall, the shock dynamics is mainly controlled by the intrinsic nonlinearity of its free propagation. The question of whether shock motion predicted by the GGSD systems as a function of time converge to the true solution for the Euler equations when the GGSD system order increases remains open until a direct comparison can be made.

\section{Numerical results}

We describe numerical simulations of the initial-value problem formulated in Sec. 4 for the specific heat ratio fixed at $\gamma=5 / 3$. Spatial derivatives with respect to $\beta$ in the GGSD systems are discretised using a fourth-order compact finite difference scheme (Lele 1992), for its ability to capture traveling waves and pseudo-spectral convergence, on a uniform grid in $\beta$ of size $N=2048$. The spectral method is not used owing to large aliasing errors produced to the high order nonlinearities in the systems. Time integration is performed with a variable time-stepping fourth-order Runge-Kutta method (RK4). All results are presented using the length unit $\tau=a_{0} M_{0} t$ for time measurement, as $\tau$ corresponds to the distance traveled by an unperturbed shock at any given time $t$.

If the shock profile remains smooth we expect that $M(\beta, t), z(\beta, t)$ will remain a singlevalued functions with bounded derivatives. We consider that a singularity has appeared at a time when an unbounded derivative in any quantity, of any order has developed. As 
the systems evolve an edge-detection algorithm (Gelb \& Tadmor 2006) that searches for discontinuities on discrete data is applied on the data set representing $M(\beta, t)$. A brief outline of the algorithm is given in Appendix D. Detection of an "edge" in the discrete $M(\beta, t)$ at some $t=t_{c}$ is interpreted to indicate the presence of an infinite $\partial M / \partial \beta$ corresponding to "overturning", and hence a singularity in the GGSD solution. This will generally also indicate the occurrence of curvature singularities in the shock shape $Z$ (Mostert et al. 2017, 2018a). For each set of given initial values, varying the choices of the two required tuning parameters $q$ and $J_{\text {crit }}$ [see Eqs. (D 7) and (D 8) in Appendix D] leads to less than $2 \%$ variations in estimating $t_{c}$ compared to the reported $t_{c}$ values obtained using $q=1.1$ and $J_{\text {crit }}=1$.

For $t>t_{c}$, the numerical solution for $M(\beta, t)$ quickly develops rapid oscillations around the jump location. The spatial derivatives, $\partial M / \partial \beta$ and $\partial^{2} M / \partial \beta^{2}$, can no longer be accurately captured by the compact finite-difference method. Proper continuation of the shock evolution for $t>t_{c}$ would require the insertion of a shock-shock into the GGSD shock-evolution description, much as in done following shock formation for the Euler equations (Eggers et al. 2017). Our present interest is evolution up to $t=t_{c}$ only and so this is not done presently. It is demonstrated in the sequel that all three GGSD systems, the zeroth, first and second order, predict spontaneous development of curvature singularities on the shock shape at a finite time that is inversely proportional to the small initial perturbation size $\epsilon$. The critical time at which singularity occurs converges within the GGSD hierarchy as the order of systems increases.

\subsection{Spontaneous singularity formation}

We first show the perturbation profiles of Mach number $m$, and shock shape $z$ as a function of the ray-tube marker $\beta$ in figure 3(a) and (b), respectively, for successive times obtained using GGSD-0 with initial piston Mach number $M_{0}=50$ and perturbation amplitude $\epsilon=0.03$. Here, curves of different times are separated by the period of the fundamental linear mode in Eq. (3.37), i.e., $\Delta \tau=1 / \sqrt{\zeta_{1}}=13.2$, up to the critical time, $t_{c}=52.7$, when discontinuities in $m$ are detected by the edge-detection algorithm. As time increases, it is seen in figure 3(a) that the symmetric $m$ distribution steepens in each oscillation cycle, and correspondingly in figure 3(b), the normal shock displacement given by $\operatorname{Im}(z)$ evolves towards the formation of two kinks. Because the lateral displacement $\operatorname{Re}(z)$ is a continuous function of $\beta$, the observed loss of continuity in $m$ and smoothness in $z$ also occur spatially in terms of shock positions $Z$. In a closer look, figure 3(c) and (d) employ a smaller time interval $\Delta \tau=0.5$ within one period for the same parameters $M_{0}=50, \epsilon=0.03$ to show leading up to the critical time, the evolution of shock slope $s$, calculated as $s=\operatorname{Im}(\partial Z / \partial \beta) / \operatorname{Re}(\partial Z / \partial \beta)$; and curvature $\kappa$, identified as $\kappa=\Phi$ thanks to Eqs. (2.22) and (3.13). We see that near the critical time, $s$ is everywhere finite but approaching a discontinuity at the point where $\kappa$ appears to form a singularity. These results are consistent with the findings of Mostert et al. (2018a), where the Whitham's original GSD equations are applied to a hypothetical initial value problem for a plane shock with a single Mach number perturbation.

Although the example given here is obtained using GGSD-0, the character of the spontaneous curvature singularity development is similarly found for GGSD-1 and GGSD-2. It is thus suggested that the "shock-shock" mechanism described by Whitham (2011), where nonlinear compression waves propagating along the shock, reinforcing each other to the formation of discontinuities, also holds in the present study where a global $A-M$ relation breaks down and non-uniform effects of the flow immediately behind the shock is accommodated.

Next, typical weak and strong shocks' evolving trajectories for $0 \leqslant \tau \leqslant \tau_{c}$, predicated 
(a)

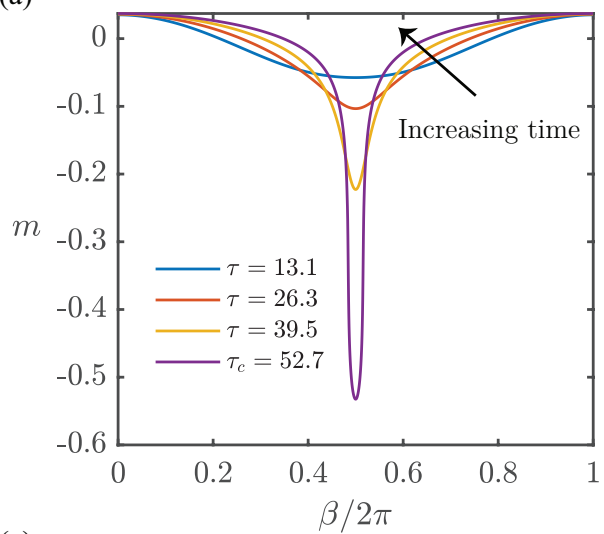

(c)

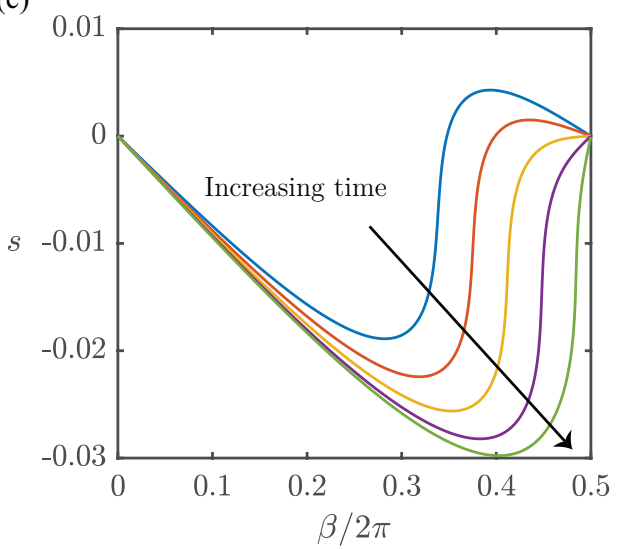

(b)

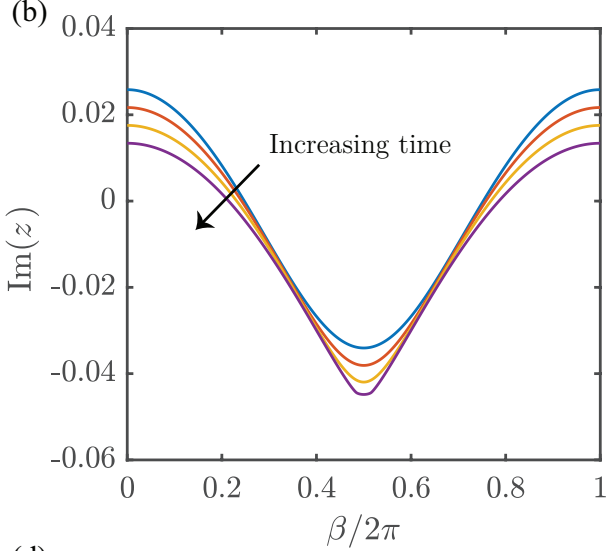

(d)

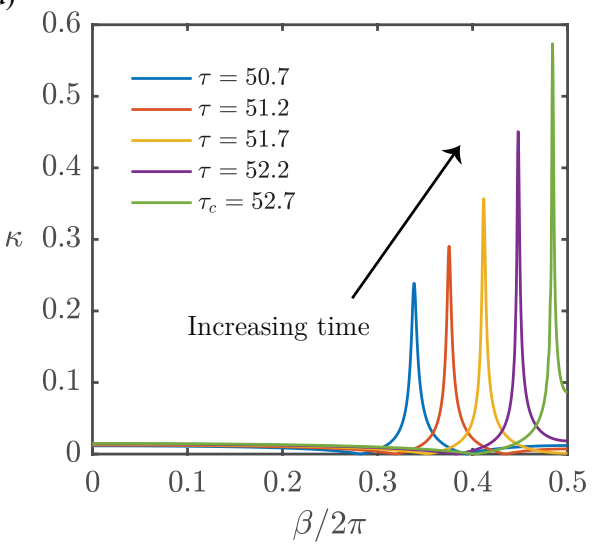

FIGURE 3. Spontaneous development of curvature singularities illustrated using GGSD-0 with initial conditions $M_{0}=50$ and $\epsilon=0.03$. The Mach number and shock shape perturbation profiles are given over four oscillation periods up to the critical time in (a) and (b), respectively. The shock slope and curvature distributions are shown as the critical time is approached with time increments of $\Delta \tau=0.5$, in (c) and (d), respectively.

by the three GGSD systems are presented in figure 4 for both a weak shock with $M_{0}=1.1$, $\epsilon=0.05[4(\mathrm{a})-(\mathrm{c})]$ and a strong shock with $M_{0}=10, \epsilon=0.05[4(\mathrm{~d})-(\mathrm{f})]$. A transition of the amplified shock shape from its smooth initial value to a non-differentiable profile with two cusps at critical time is observed in all cases shown. The oscillatory shock shape patterns given by GGSD-0 is evident in both limits, with oscillation frequencies that match the linear analysis of fundamental mode in Eq. (3.37), i.e., $\omega=\sqrt{\zeta_{1}}$ in the strong shock limit $\left(M_{0}=10\right)$ and $\omega=\sqrt{\left(M_{0}-1\right) / 2}$ in the weak shock limit $\left(M_{0}=1.1\right)$. Comparing the GGSD models of different orders, differences in the detailed trajectories are small for $M_{0}=1.1$, noting that the values for $\tau_{c}$ are also close to each other. This suggests that in the weak shock limit, the non-uniform effect behind the shock is small and that GGSD-0 provides a good approximation.

In contrast, with $M_{0}=10$, the first and second order systems predict very similar trajectories with almost the same $\tau_{c}$ that is significantly shorter than the zeroth-order value. In this case, evolution of the Mach number perturbation $m$ is further investigated in figure 5 using the same parameters $M_{0}=10$ and $\epsilon=0.05$, where the profiles of $m$ along the shock are shown for the entire history of the shock trajectories. The nonlinear solution of GGSD-0 seen in figure 5(a) again shows the oscillatory pattern that 

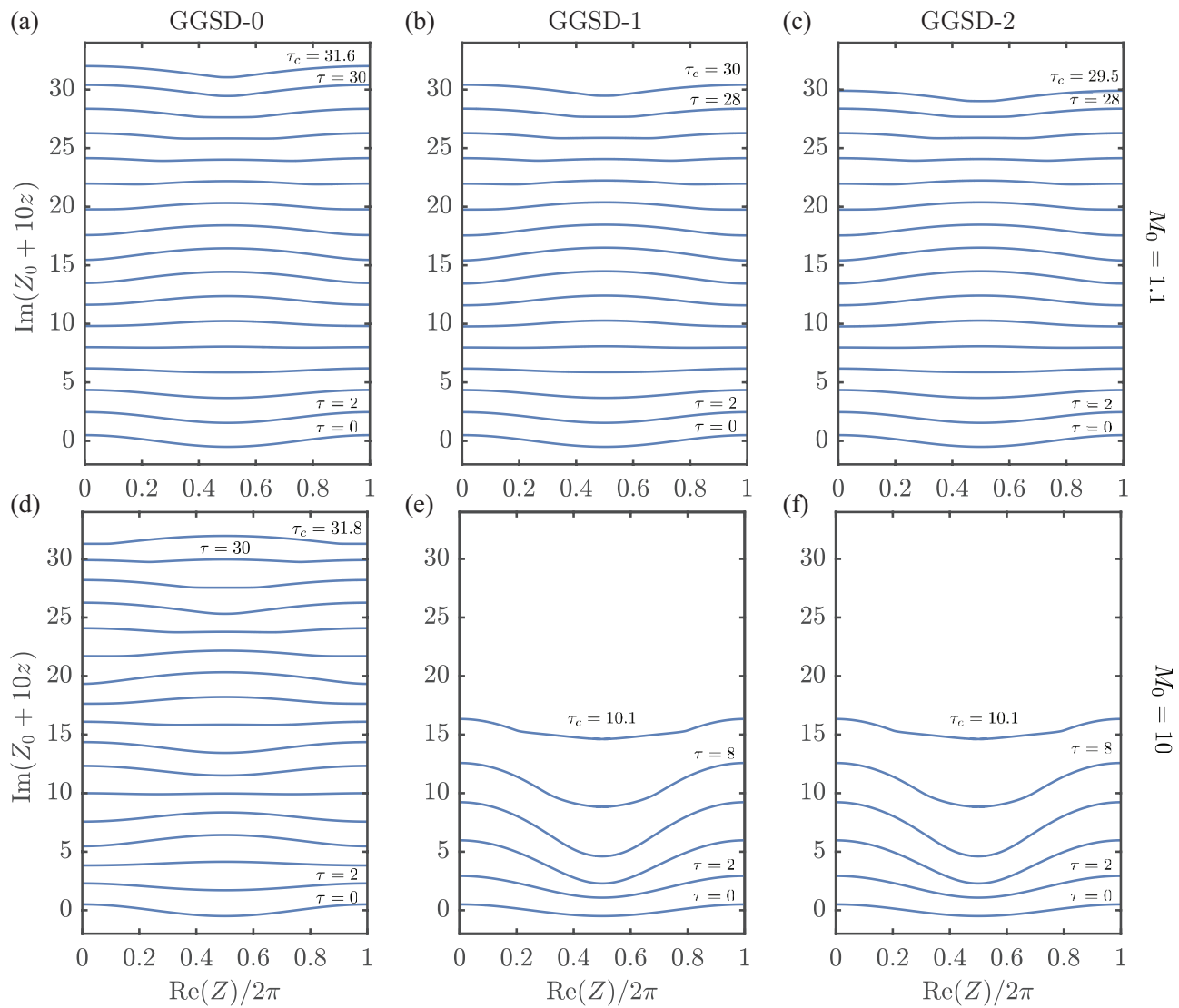

FiguRE 4. Shock trajectories with perturbation in the $Y$-direction amplified ten times for piston perturbation size $\epsilon=0.05$. The initial piston Mach number is $M_{0}=1.1$ in (a)-(c) and $M_{0}=10$ in (d)-(f), corresponding to results obtained from the zeroth, first and second order GGSD systems, respectively. In each panel, successive trajectories are shown in increments of $\Delta \tau=2$, up to the final curve given at the critical time $\tau_{c}$.

is consistent with the corresponding linear theory, noting that stable oscillation in time is here terminated by the onset of discontinuities in $m$ owing to reinforcing waves on the shock. In contrast, for GGSD-1 and GGSD-2 where linear analysis predicts a growth that is linear in time, the Mach number profiles in figure 5(b) and (c) demonstrate that the underlying osculations are dominated by approximately linear growth, until nonlinear effects accumulate, leading to $m$-discontinuities at the critical time. This result shows that the initial and subsequent downstream non-uniformity plays a crucial role in determining the shock motion.

\subsection{Critical time}

Figure 6 shows $\tau_{c}$ given by the three GGSD systems as a function of the piston perturbation $\epsilon$, in both strong [figure $6(\mathrm{a}, \mathrm{b})$ ] and weak [figure $6(\mathrm{c}, \mathrm{d})$ ] shock limits. First, in agreement with the observation made from figure 4, the strong shock limit sees a dramatic reduction in $\tau_{c}$ comparing GGSD-0 to the other two higher order models, while GGSD-1 and GGSD-2 appear to have converged within the GGSD framework in terms of their $\tau_{c}$ predictions. The weak-shock limit shows much smaller differences between the three models. When at least the $Q_{1}$ correction is considered in the equations, for the 
(a)

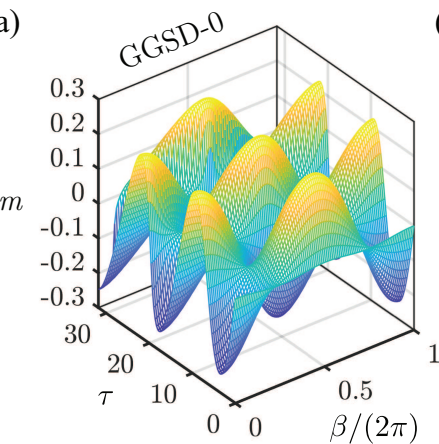

(b)

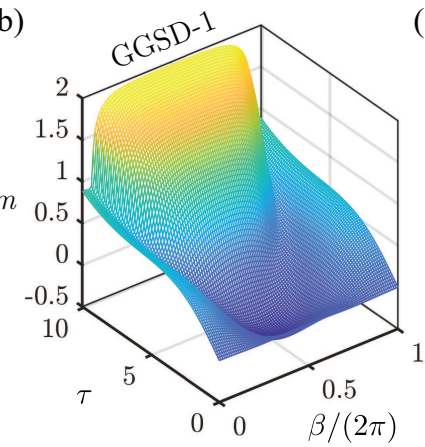

(c)

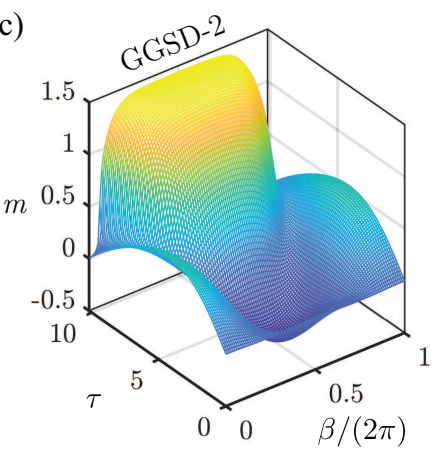

FiguRE 5. History of Mach number perturbation profile $m$ along the evolving shock, for $\epsilon=0.05$ and $M_{0}=10$. Results obtained using three different orders of GGSD models are shown in (a)-(c).

cases shown here with initial Mach number $M_{0}=10$ and $M_{0}=50$, the decrease in $\tau_{c}$ is found to be over $50 \%$ across all simulations with varying $\epsilon$. For $M_{0}=1.1$ and $M_{0}=1.02$ a decrease in $\tau_{c}$ is still consistently present, although the magnitude falls within the uncertainties of the edge-detection algorithm. On the other hand, the difference made by further including the $Q_{2}$ correction in GGSD-2 is insignificant for all $M_{0}$ simulated. These results provide convincing evidence for the convergence of the GGSD hierarchy and showcase the power of higher-order methods in handling shocks that lead to a strongly non-uniform flow, whose effect seems to increase with Mach number.

An intuitive explanation of the GGSD hierarchy convergence in this application can be offered by comparing the order of magnitude estimates made for $Q_{1}$ and $Q_{2}$ when deriving the GGSD equations, to the independently calculated initial values of $Q_{1}$ and $Q_{2}$. In Sections 3.2 and 3.3, it was shown that $Q_{1} \gtrsim O\left(\varepsilon_{\mathrm{w}}^{0}\right), Q_{2} \gtrsim O\left(1 / \varepsilon_{\mathrm{w}}\right)$ as $\varepsilon_{\mathrm{w}}=M-1 \rightarrow 0$, and $Q_{1} \gtrsim O\left(M^{3}\right), Q_{2} \gtrsim O\left(M^{4}\right)$ as $M \rightarrow \infty$, in order to retain the corresponding $Q_{1}$ and $Q_{2}$ corrections in the leading order dominant balance. Meanwhile, as $\epsilon \rightarrow 0$, the initial conditions for $Q_{1}$ and $Q_{2}$, obtained in Sec. 4.3 without the GGSD truncation error, are $Q_{1} \sim \epsilon\left(M_{0}-1\right), Q_{2} \sim \epsilon^{2}\left(M_{0}-1\right)^{3}$ as $M_{0} \rightarrow 1$ and $Q_{1} \sim \epsilon M_{0}^{3}, Q_{2} \sim \epsilon^{2} M_{0}^{4}$, as $M_{0} \rightarrow \infty$. As a result, the initial condition that best matches its minimum order of magnitude required by the asymptotic GGSD equations is that of $Q_{1}$ in the strong shock limit, where indeed the effect of $Q_{1}$ on $\tau_{c}$ is shown the largest.

Another finding of interest from figure 6 is that all three GGSD methods show a critical time $\tau_{c}(\epsilon)$ as inversely proportional to the initial perturbation size $\epsilon$ for sufficiently small $\epsilon$, for all Mach numbers $M_{0}$. A suggested form is

$$
\tau_{c}=\tau_{c}\left(\epsilon, M_{0} ; d\right)=\frac{K_{1}\left(M_{0} ; d\right)}{\epsilon}, \quad \epsilon \ll 1,
$$

where $K_{1}$ is a proportionality constant that depends on $M_{0}$ and the order of the GGSD models, $d \in\{0,1,2\}$. The asymptotic domain of minimum $\epsilon$ where (5.1) applies seems to be $M_{0}$ dependent, particularly for strong shocks. Together with the similar analytic form obtained by Mostert et al. (2018a), the present results, including the established connection between the GGSD hierarchy and the Euler equations, provides compelling evidence that (5.1) is a universal form for perturbed planar shock waves.

From a computational perspective we note that in figure 6 , the smallest $\epsilon$ achieved in the strong shock limit is $\epsilon=0.002$, doubling $\epsilon=0.001$ in the weak shock limit. This is because an explicit time integration method such as RK4 appears to be unstable and costly for further reduction in $\epsilon$. An implicit method might be preferred should results 
(a)

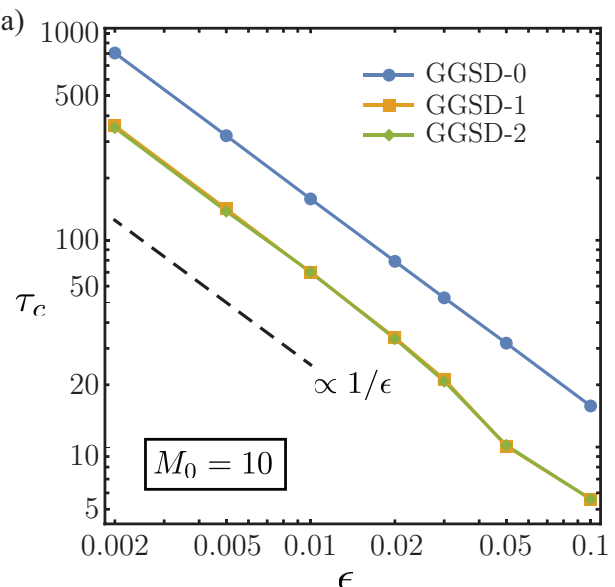

(c)

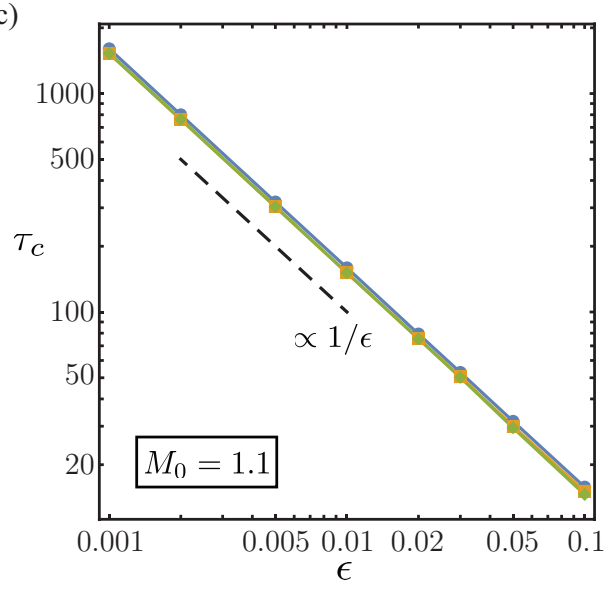

(b)

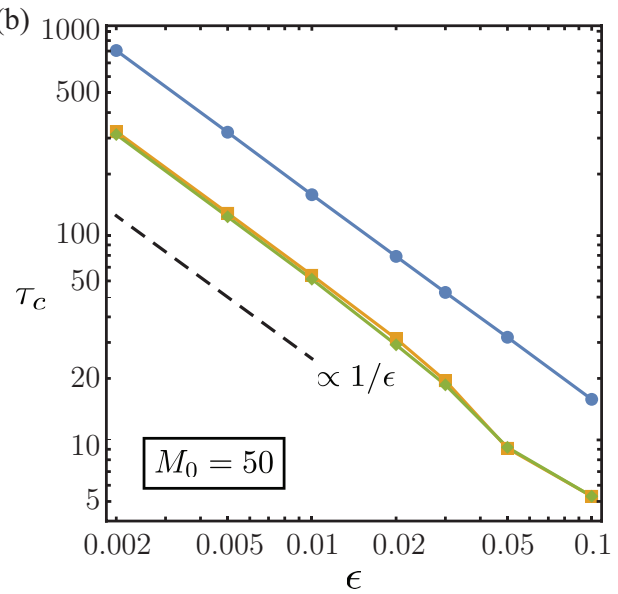

(d)

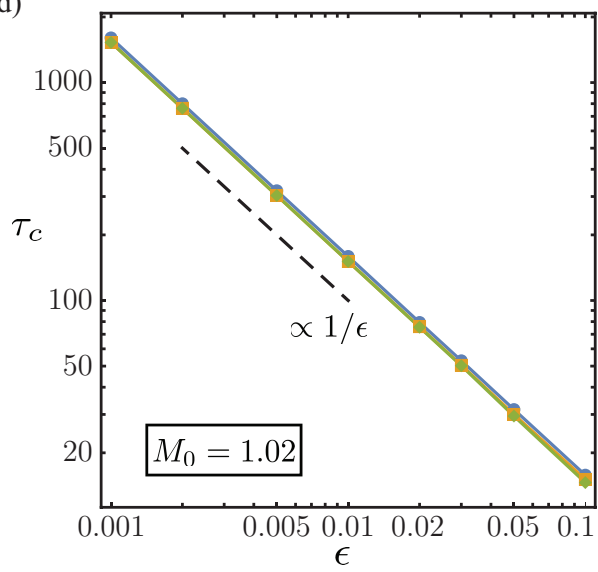

Figure 6. Critical times in strong and weak shock limits given for (a) $M_{0}=10$, (b) $M_{0}=50$, (c) $M_{0}=1.1$ and (d) $M_{0}=1.02$. In all four cases, results obtained using GGSD-0 are shown as disks, GGSD-1 as squares and GGSD-2 as diamonds. In each panel, the dashed line without markers displays a reference function that has inverse power law of degree one.

for $\epsilon<0.002$ and $M \gg 1$ be desired. This is not done presently owing to the associated high cost of iteratively solving order $N$ nonlinear equations at each time step.

To illustrate that the choice of $M_{0}$ in figure 6 adequately represent the strong and weak shock limits, figure 7 plots $\tau_{c}$ for two sequences of extreme values of $M_{0}$, obtained for $\epsilon=0.02$ when $M_{0} \gg 1$ and $\epsilon=0.01$ when $M_{0}-1 \ll 1$, in all three GGSD systems. Convergence in $\tau_{c}$ is clear as $M_{0}>50$ and $M_{0}-1<0.02$ for strong and weak shocks, respectively.

\subsection{Diagnostic statistics}

We have seen in figure 4 that the GGSD-1 and GGSD-2 in the strong shock limit produce distinctly interesting shock trajectories before curvature singularities form. Figure 8 therefore presents the key statistics that characterises the evolution given by these higher order methods up to their critical times using $M_{0}=50$ and $\epsilon=0.02$. The mean and standard deviation of Mach number perturbation over the shock, defined as

$$
\bar{m}=\frac{1}{2 \pi} \int_{0}^{2 \pi} m d \beta, \quad \sigma(m)=\left(\frac{1}{2 \pi} \int_{0}^{2 \pi}(m-\bar{m}) d \beta\right)^{1 / 2},
$$


(a)

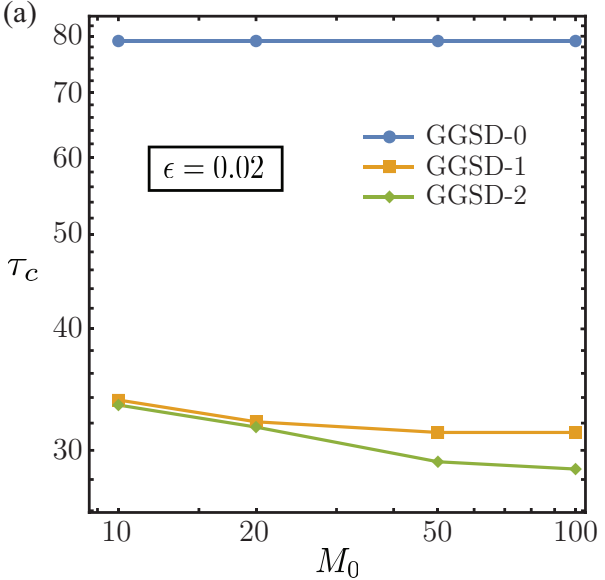

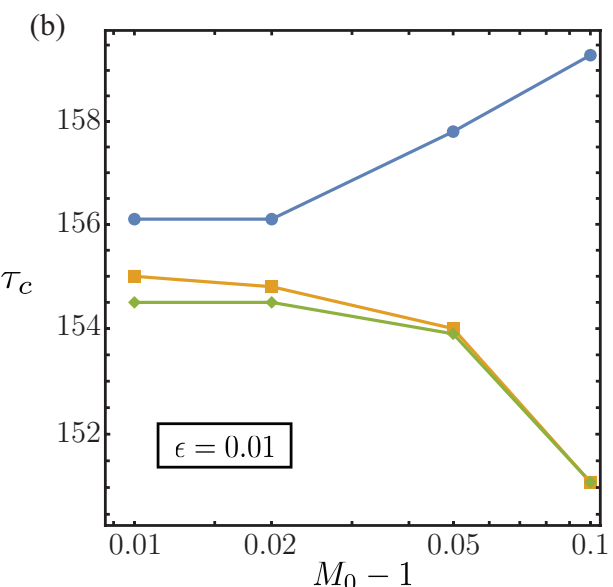

Figure 7 . Convergence of critical time as $M_{0}$ increases in the strong shock limit for $\epsilon=0.02$ in (a), and as $M_{0}$ decreases in the weak shock limit for $\epsilon=0.01$ in (b). Disks, squares and diamonds correspond to GGSD-0, GGSD-1 and GGSD-2 data.

(a)

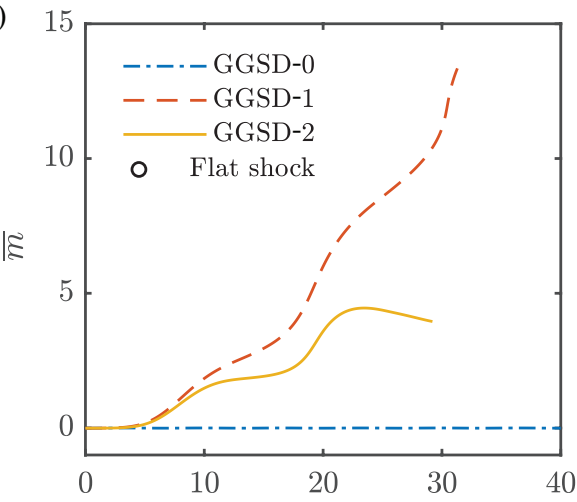

(c)

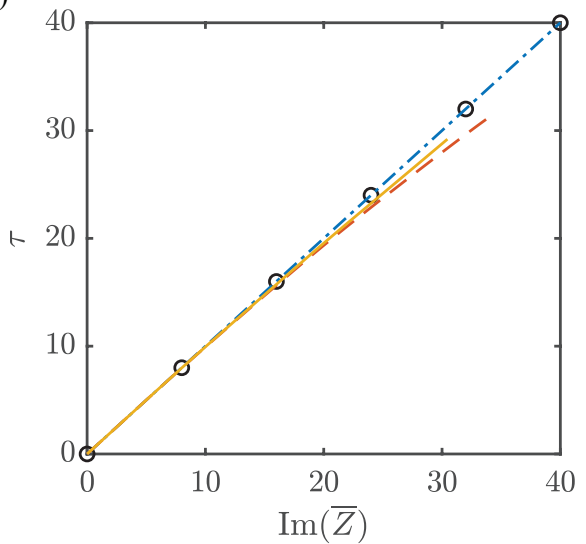

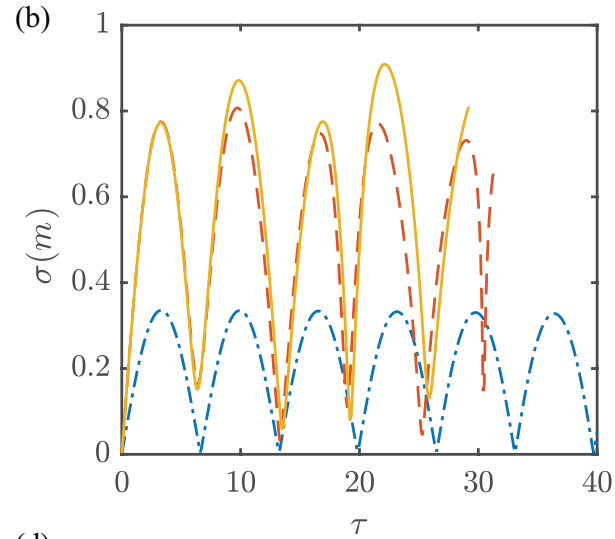

(d)

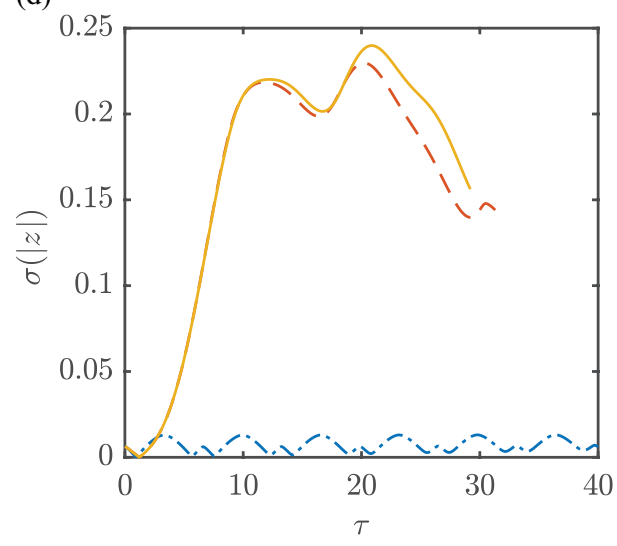

Figure 8. Temporal shock profile statistics comparison between the flat (hollow circles) and perturbed shock due to three GGSD systems (lines of different dashing), made for $M_{0}=50$ and $\epsilon=0.02$. (a,b) Mean and standard deviation of the Mach number perturbation profile. (c) Average shock advancement in the unperturbed shock normal direction. (d) Standard deviation of the shock shape perturbation. 
are shown in figure 8 (a) and (b), respectively. It is observed that GGSD-0 gives an oscillating Mach number perturbation profile around the mean, that is zero, across the profile, for all time until a discontinuity develops (critical time not shown for the dotted dash lines in the figures). GGSD-1 and GGSD-2 both produce on average an upward drift for $m$ over time, where the increase is smaller with GGSD-2. This is consistent with the linear analysis in Sec. 3.5, where Eqs. (3.33) and (3.38) clearly states the linear growth. Whether this mean drift will further reduce with increasing order of the GGSD systems, or if it faithfully represents the Euler equations, remains to be confirmed by direct comparisons against direct numerical simulations of the full Euler equations with high-order accurate shock tracking. Nevertheless, despite the mean rise, the standard deviation of $m$ obtained from GGSD-1 and GGSD-2 shows qualitatively similar periodic fluctuations that do not grow in times, as seen in GGSD-0.

Further, as a consequence of the positive Mach number perturbation increasing, the shock trajectories given by the higher order methods must be accelerating at an increasingly faster rate compared to either the unperturbed flat shock, or the GGSD-0 prediction. Indeed, this is reflected in the figure $8(\mathrm{c})$ where the mean shock advancement, $\operatorname{Im}(\bar{Z})$, is plotted as a function of $\tau$. As expected from $\bar{m}=0$, the zeroth order GGSD system yields a history of mean shock positions that is exactly the same as the unperturbed shock; whereas with an increasing positive $\bar{m}$, the mean shock position given by GGSD-1 and GGSD-2 accelerate and outrun the flat shock. Particularly, the $Q_{2}$ correction made by GGSD-2 to GGSD-1 partially negates the $Q_{1}$ correction applied by GGSD-1 to GGSD-0. Additionally, figure 8(d) shows that although the standard deviation $\sigma(|z|)$, defined analogously to Eq. (5.2), is significantly larger in GGSD-1 and GGSD-2 compared to GGSD-0, the shock shape perturbations remain bounded.

The negation effect of GGSD-2 is related to the measure of immediate non-uniformity $Q_{1}$ shown in figure 9 , as a function of the evolving shock position in the complex plane for the same parameters $M_{0}=50$ and $\epsilon=0.02$. Identically zero in GGSD-0, the evolution of non-zero $Q_{1}$ in the higher-order models has been demonstrated to provide a considerable Mach number correction. Specifically, comparing figure 9(a) and (b), the overall larger increase of $Q_{1}$ given by GGSD-1 than GGSD-2, especially the fact that when the critical time is approached $Q_{1}$ actually decreases in GGSD-2, matches the different growth rates of $\bar{m}$ observed in figure $8(\mathrm{a})$.

Curiously, referencing figure $8(\mathrm{~b})$, the distance gap between mean shock position predicted by GGSD-1 and GGSD-2 is further examined in figure 10. Denoting $\Delta Y$ as the lead distance, its maximum obviously occurs at the critical time of each model. The percentage of $\max (\Delta Y)$ over the advancement of the reference flat shock, which also equals $\tau_{c}$ by definition, is shown in figure 10(a) as a function of $\epsilon$. A converging result of approximately $9.5 \%$ for GGSD-1 and $4.5 \%$ for GGSD-2 is found, noting that $\max (\Delta Y) / \tau_{c}$ is overall weakly dependent on the simulated range of $\epsilon$. More interestingly, examining $\Delta Y$ at a given time $\tau$ that is at most the earliest critical time among different models, i.e., $\tau<\min _{d \in\{0,1,2\}}\left(\tau_{c}\left(M_{0} ; d\right)\right)$, for instance choosing $\tau=\tau_{c}(0.03,50 ; 2)=18.6$ as shown in figure $10(\mathrm{~b})$, reveals a quadratic scaling law with respect to $\epsilon$ which holds for both GGSD-1 and GGSD-2, that is,

$$
\left.\Delta Y\right|_{\tau}=K_{2}\left(M_{0} ; d\right) \epsilon^{2},
$$

where again $K_{2}$ is the constant multiplier that depends on $M_{0}$ and order number $d \in$ $\{1,2\}$.

In order to enable such distance lead obtained using higher order methods over a flat shock, the flow behind the shock must gain more kinetic energy from the corrugated piston advancing, than for the flat piston flow. Unlike a flat piston whose motion is given 
(a)

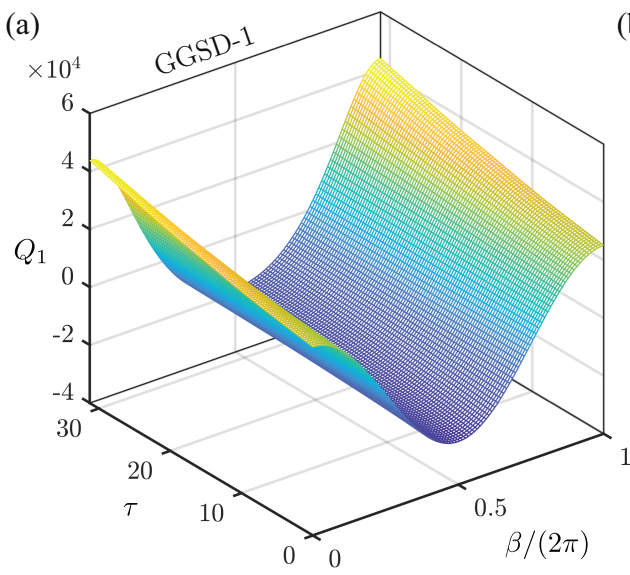

(b)

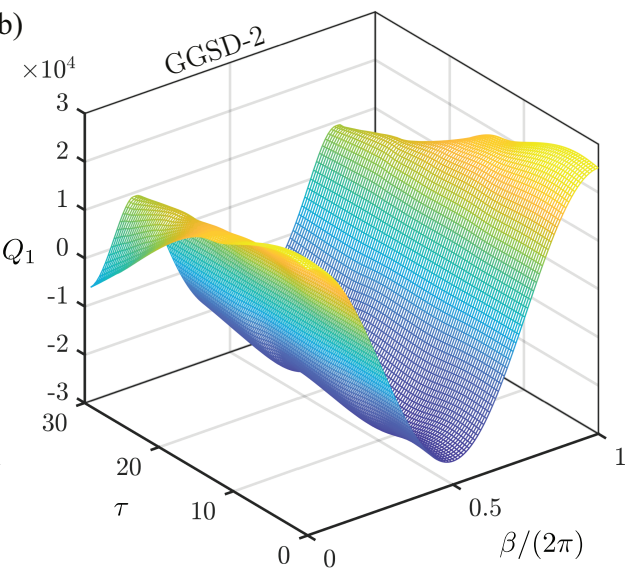

FiguRE 9 . History of the non-uniformity measure $Q_{1}$ behind the evolving shock as a function of shock positions in two-dimensions. (a) and (b) compare results obtained for $M_{0}=50$ and $\epsilon=0.02$, using GGSD-1 and GGSD-2, respectively.

(a)

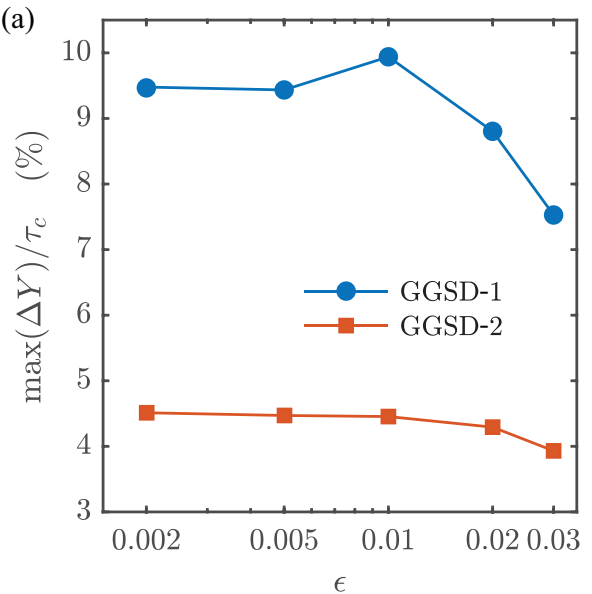

(b)

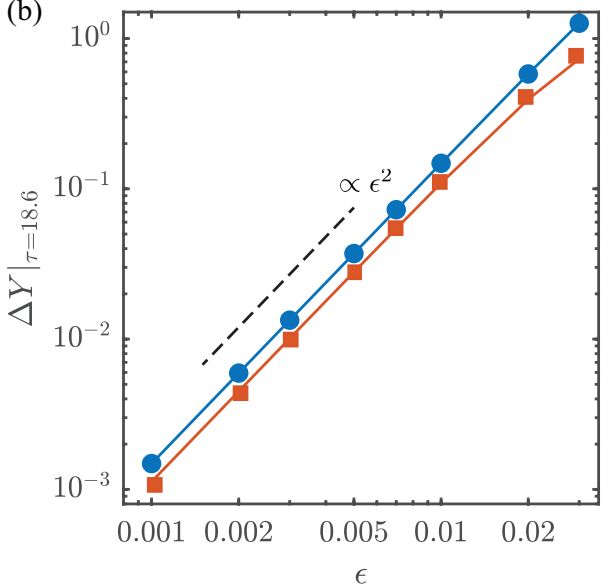

Figure 10. (a) Percentage maximum distance lead of average shock position given by GGSD-1 (disks) and GGSD-2 (squares) over the reference flat shock. (b) Distance lead of average shock position at a fixed time that is the critical time for the second order method. In both cases, $M_{0}=50, \epsilon=0.03$, and the dashed line represents the first order, while the solid line gives the second order method. The dashed line without markers in (b) shows an explicit function of quadratic power law.

by the Heaviside function [see Eq. (4.1)], which advances at the same speed with the flow behind the shock for all $t>0$, a corrugated piston of the same velocity continues to accelerate fluid in the local normal direction, possibly generating more kinetic energy into the flow for $t>0$.

\section{Conclusions}

We have reported numerical simulations of the evolution of shock produced by an impulsively accelerated, two-dimensional piston with a sinusoidal surface corrugation of amplitude $\epsilon$. First a complex-variable formulation of generalised geometrical shock dynamics, referred to as GGSD, is derived up to second order for both strong- and weak- 
shock limits. The resulting closed systems of partial differential equations are then solved numerically to give the evolution of the shock shape and Mach number distributions. An edge-detection algorithm is applied to test for the appearance of a singularity in the shock evolution profile at some critical time $\tau_{c}$. These simulations provide strong evidence supporting the hypothesis that a spontaneous curvature singularity develops in the shock shape as a prelude to the formation of triple points or Mach stems in $\tau_{c}$ inversely proportional to the amplitude of the piston corrugation.

The adoption of the GGSD hierarchy overcomes the limitations of previously used theories of geometrical shock dynamics by first accommodating well-defined shock initial conditions for the shock generation process, which are calculated exactly for the pistondriven shock according to the Euler equations, and second, by incorporating a quantitative description of non-uniform flow effects immediately behind the shock. The behaviour of weak shocks with Mach number close to unity are found to witness small changes when increasing the system order, while maintaining oscillations around the unperturbed shock up to the critical time of curvature singularity formation. For strong shocks with large Mach number, the inclusion of non-uniform flow correction $Q_{1} \neq 0$ behind the shock in GGSD-1 and GGSD-2 predicts an overall shock acceleration compared to GGSD-0 where $Q_{1}=0$. The corresponding Mach number perturbation growth is eventually terminated by the onset of the curvature singularity, which occurs in GGSD-1 and GGSD-2 at $\tau_{c}$ that converges with the GGSD order increasing, and that scales as $\tau_{c} \propto 1 / \epsilon$ as in GGSD-0 albeit with a significantly smaller proportionality constant. Between the higherorder methods, GGSD-2 gives a smaller $Q_{1}$ correction effect than GGSD-1, manifested particularly in the smaller average shock acceleration relative to the unperturbed shock. This leaves uncertainty in the convergence of shock trajectories given by the GGSD expansion hierarchy, and remains to be resolved by direct numerical simulations for the Euler equations with accurate shock tracking.

\section{Acknowledgement}

This work was supported by the KAUST Office of Sponsored Research under Award No. URF/1/3418-01. The authors also thank Professor H. G. Hornung for helpful discussions.

\section{Declaration of Interests}

The authors report no conflict of interest.

\section{Appendix A. Tables of coefficients}

This section lists all the coefficient functions used throughout this study in Table 1 and 2 , as constant functions of the specific heat ratio $\gamma$. 


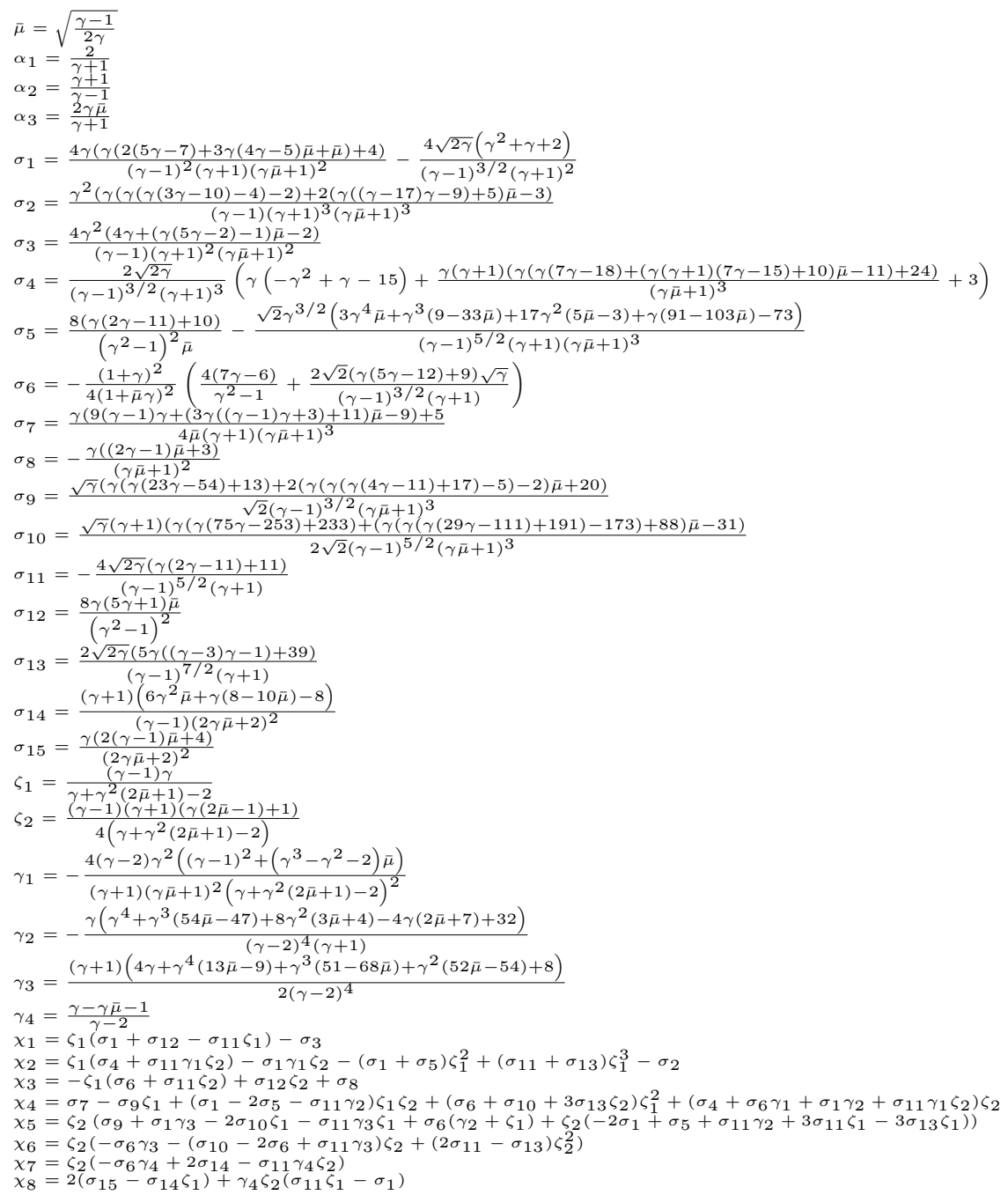

TABLE 1. Constant coefficient functions in the GGSD systems, up to second order, in terms of the specific heat ratio $\gamma$. 


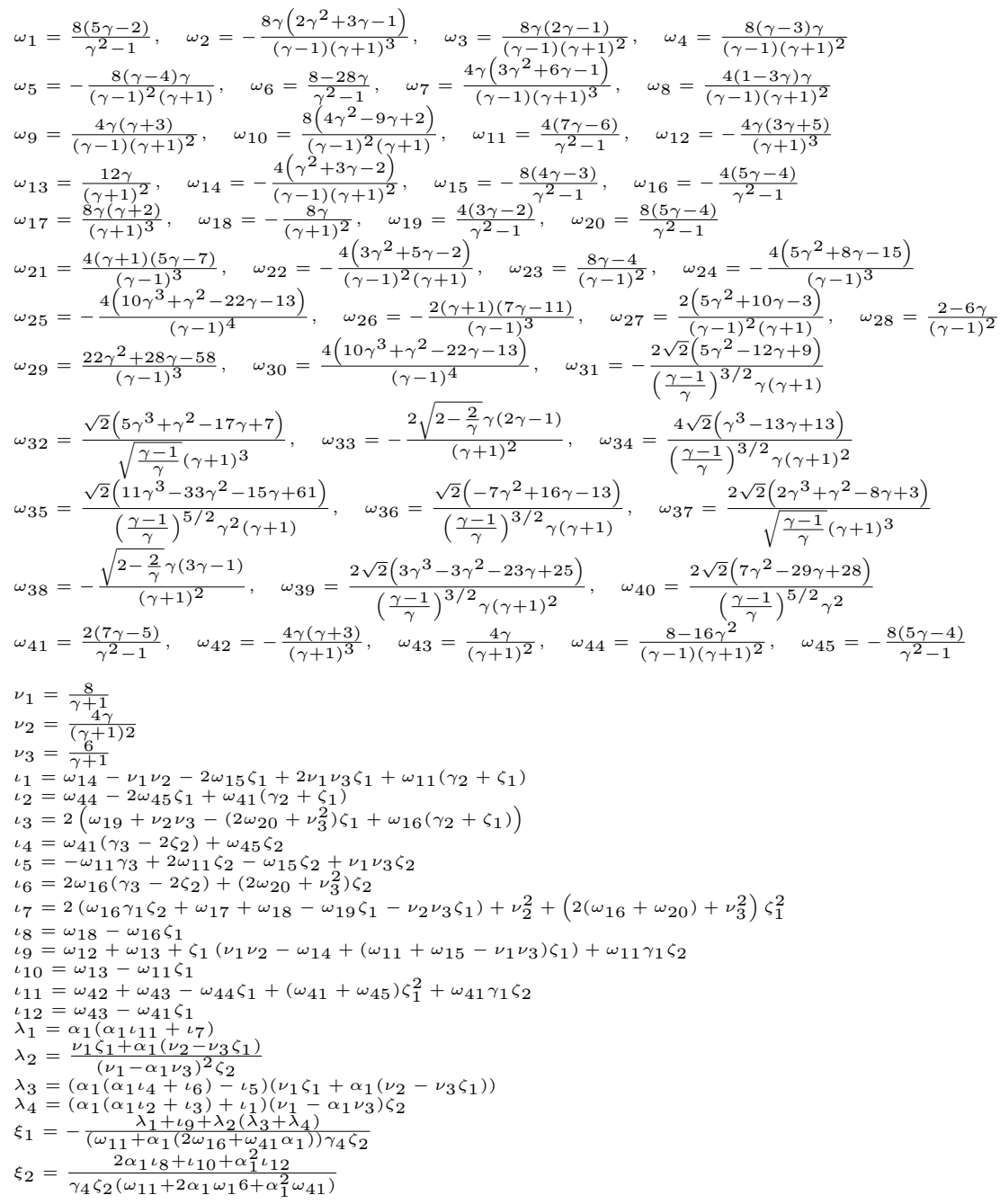

TABle 2. Constant coefficients for the second order partial derivatives of flow variables in the strong shock limit, found in Eqs. (B 5)-(B 8); and those for the initial value of $Q_{2}$, found in Eq. (C 1), associated with shock driven by a corrugated piston. These coefficients are expressed in terms of other constant functions of $\gamma$ listed in Table 1. 


\section{Appendix B. Partial derivatives of flow variables}

The partial derivatives used in the expansions of GGSD systems are given in this sections. Asymptotic expressions of the general solutions to Eq. (2.28) are obtained for both the weak and strong shock limits up to second order.

In the weak shock limit where $\varepsilon_{\mathrm{w}}=M-1 \rightarrow 0$, and the strong shock limit where $M \sim M^{\prime} \rightarrow \infty$, the first order partial derivatives are given by

$$
\begin{aligned}
& \rho_{t}= \begin{cases}\frac{Q_{1}}{2 a_{0}^{2}}+2\left[\frac{2 Q_{1}}{(1+\gamma) a_{0}^{2}}+\frac{(\gamma-3) \rho_{0} a_{0} \Phi}{(1+\gamma)^{2}}\right] \varepsilon_{\mathrm{w}}+O\left(\varepsilon_{\mathrm{w}}^{2}\right), & \varepsilon_{\mathrm{w}} \rightarrow 0, \\
\rho_{0} a_{0}\left[\frac{6(\gamma+1)}{(\gamma-1)^{2}} M^{\prime}+\frac{2}{\gamma-1} \Phi M\right]+O\left(M^{0}\right), & M \rightarrow \infty,\end{cases} \\
& \rho_{x}= \begin{cases}\frac{-Q_{1}}{2 a_{0}^{3}}+\left[\frac{(\gamma-3) Q_{1}}{(1+\gamma) a_{0}^{3}}-\frac{4(\gamma-1) \rho_{0} \Phi}{(1+\gamma)^{2}}\right] \varepsilon_{\mathrm{w}}+O\left(\varepsilon_{\mathrm{w}}^{2}\right), & \varepsilon_{\mathrm{w}} \rightarrow 0, \\
-\rho_{0}\left[\frac{6(\gamma+1)}{(\gamma-1)^{2}} \frac{M^{\prime}}{M}+\frac{2}{\gamma-1} \Phi\right]+O\left(M^{-1}\right), & M \rightarrow \infty,\end{cases} \\
& u_{t}= \begin{cases}\frac{Q_{1}}{2 \rho_{0} a_{0}}+\left[\frac{Q_{1}}{\rho_{0} a_{0}}+\frac{4(\gamma-1) a_{0}^{2} \Phi}{(1+\gamma)^{2}}\right] \varepsilon_{\mathrm{w}}+O\left(\varepsilon_{\mathrm{w}}^{2}\right), & \varepsilon_{\mathrm{w}} \rightarrow 0, \\
a_{0}^{2} M\left[\frac{8}{\gamma+1} M^{\prime}+\frac{4 \gamma}{(\gamma+1)^{2}} \Phi M\right]+O\left(M^{1}\right), & M \rightarrow \infty,\end{cases} \\
& u_{x}= \begin{cases}\frac{-Q_{1}}{2 \rho_{0} a_{0}^{2}}+\left[\frac{(2-6 \gamma) a_{0} \Phi}{(1+\gamma)^{2}}\right] \varepsilon_{\mathrm{w}}+O\left(\varepsilon_{\mathrm{w}}^{2}\right), & \varepsilon_{\mathrm{w}} \rightarrow 0, \\
-a_{0}\left[\frac{6}{\gamma+1} M^{\prime}+\frac{4 \gamma}{(\gamma+1)^{2}} \Phi M\right]+O\left(M^{0}\right), & M \rightarrow \infty,\end{cases} \\
& p_{t}= \begin{cases}\frac{Q_{1}}{2}+2\left[Q_{1}+\frac{(\gamma-3) \rho_{0} a_{0}^{3} \Phi}{(1+\gamma)^{2}}\right] \varepsilon_{\mathrm{w}}+O\left(\varepsilon_{\mathrm{w}}^{2}\right), & \varepsilon_{\mathrm{w}} \rightarrow 0, \\
\rho_{0} a_{0}^{3} M^{2}\left[\frac{4(3 \gamma-2)}{\gamma^{2}-1} M^{\prime}+\frac{4 \gamma}{(\gamma+1)^{2}} \Phi M\right]+O\left(M^{2}\right), & M \rightarrow \infty,\end{cases} \\
& p_{x}= \begin{cases}\frac{-Q_{1}}{2 a_{0}}-\left[\frac{Q_{1}}{a_{0}}+\frac{4(\gamma-1) \rho_{0} a_{0}^{2} \Phi}{(1+\gamma)^{2}}\right] \varepsilon_{\mathrm{w}}+O\left(\varepsilon_{\mathrm{w}}^{2}\right), & \varepsilon_{\mathrm{w}} \rightarrow 0, \\
-4 \rho_{0} a_{0}^{2} M\left[\frac{2 \gamma-1}{\gamma^{2}-1} M^{\prime}+\frac{\gamma}{(\gamma+1)^{2}} \Phi M\right]+O\left(M^{1}\right), & M \rightarrow \infty,\end{cases}
\end{aligned}
$$

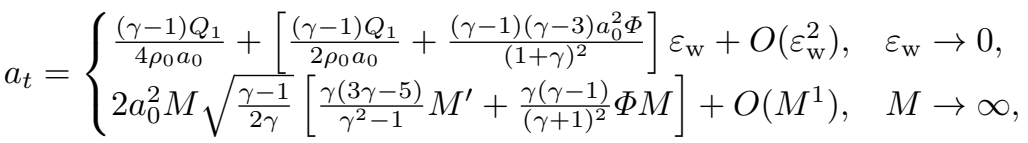

$$
\begin{aligned}
& a_{x}= \begin{cases}\frac{-(\gamma-1) Q_{1}}{4 \rho_{0} a_{0}^{2}}-\left[\frac{2(\gamma-1)^{2} a_{0} \Phi}{(1+\gamma)^{2}}\right] \varepsilon_{\mathrm{w}}+O\left(\varepsilon_{\mathrm{w}}^{2}\right), & \varepsilon_{\mathrm{w}} \rightarrow 0, \\
-a_{0} \sqrt{\frac{\gamma-1}{2 \gamma}}\left[\frac{4 \gamma(\gamma-2)}{\gamma^{2}-1} M^{\prime}+\frac{2 \gamma(\gamma-1)}{(\gamma+1)^{2}} \Phi M\right]+O\left(M^{0}\right), & M \rightarrow \infty .\end{cases}
\end{aligned}
$$

Similarly the limiting second order partial derivatives as $\varepsilon_{\mathrm{w}} \rightarrow 0$ and $M \sim M^{\prime} \sim$ $M^{\prime \prime} \rightarrow \infty$ are as follows,

$$
\begin{aligned}
& p_{t t}=\left\{\begin{array}{l}
\frac{Q_{2}}{2}+O\left(\varepsilon_{\mathrm{w}}^{0}\right), \\
\rho_{0} a_{0}^{4} M^{2}\left(M\left(\omega_{1} M^{\prime \prime}+\left(\omega_{2}+\omega_{3}\right) \Phi^{2} M+\omega_{3} \Psi M\right)+\omega_{4} \Phi M M^{\prime}+\omega_{5} M^{\prime 2}\right)+O\left(M^{3}\right),
\end{array}\right. \\
& p_{t x}=\left\{\begin{array}{l}
-\frac{Q_{2}}{2 a_{0}}+O\left(\varepsilon_{\mathrm{w}}^{0}\right), \\
\rho_{0} a_{0}^{3} M\left(M\left(\omega_{6} M^{\prime \prime}+\left(\omega_{7}+\omega_{8}\right) \Phi^{2} M+\omega_{8} \Psi M\right)+\omega_{9} \Phi M M^{\prime}+\omega_{10} M^{\prime 2}\right)+O\left(M^{2}\right)
\end{array}\right.
\end{aligned}
$$


$u_{t t}=\left\{\begin{array}{l}\frac{Q_{2}}{2 \rho_{0} a_{0}}+O\left(\varepsilon_{\mathrm{w}}^{0}\right), \\ a_{0}^{3} M\left(\omega_{11} M M^{\prime \prime}+M^{2}\left(\left(\omega_{12}+\omega_{13}\right) \Phi^{2}+\omega_{13} \Psi\right)+\omega_{14} \Phi M M^{\prime}+\omega_{15} M^{\prime 2}\right)+O\left(M^{2}\right),\end{array}\right.$

$u_{t x}=\left\{\begin{array}{l}-\frac{Q_{2}}{2 \rho_{0} a_{0}^{2}}+O\left(\varepsilon_{\mathrm{w}}^{0}\right) \\ a_{0}^{2}\left(\omega_{16} M M^{\prime \prime}+M^{2}\left(\left(\omega_{17}+\omega_{18}\right) \Phi^{2}+\omega_{18} \Psi\right)+\omega_{19} \Phi M M^{\prime}+\omega_{20} M^{\prime 2}\right)+O(M)\end{array}\right.$

$u_{x x}=\left\{\begin{array}{l}\frac{Q_{2}}{2 \rho_{0} a_{0}^{3}}+O\left(\varepsilon_{\mathrm{w}}^{0}\right), \\ \frac{a_{0}}{M}\left(\omega_{41} M M^{\prime \prime}+M^{2}\left(\left(\omega_{42}+\omega_{43}\right) \Phi^{2}+\omega_{43} \Psi\right)+\omega_{44} \Phi M M^{\prime}+\omega_{45} M^{\prime 2}\right)+O\left(M^{0}\right),\end{array}\right.$

$\rho_{t t}=\left\{\begin{array}{l}\frac{Q_{2}}{2 a_{0}^{2}}+O\left(\varepsilon_{\mathrm{w}}^{0}\right), \\ \rho_{0} a_{0}^{2}\left(\omega_{21} M M^{\prime \prime}+M^{2}\left(\left(\omega_{22}+\omega_{23}\right) \Phi^{2}+\omega_{23} \Psi\right)+\omega_{24} \Phi M M^{\prime}+\omega_{25} M^{\prime 2}\right)+O(M),\end{array}\right.$

$\rho_{t x}=\left\{\begin{array}{l}-\frac{Q_{2}}{2 a_{0}^{3}}+O\left(\varepsilon_{\mathrm{w}}^{0}\right), \\ \frac{\rho_{0} a_{0}}{M}\left(\omega_{26} M M^{\prime \prime}+M^{2}\left(\left(\omega_{27}+\omega_{28}\right) \Phi^{2}+\omega_{28} \Psi\right)+\omega_{29} \Phi M M^{\prime}+\omega_{30} M^{\prime 2}\right)+O(M),\end{array}\right.$

$a_{t t}=\left\{\begin{array}{l}\frac{(\gamma-1) Q_{2}}{4 \rho_{0} a_{0}}+O\left(\varepsilon_{\mathrm{w}}^{0}\right), \\ -a_{0}^{3} M\left(\omega_{31} M M^{\prime \prime}+M^{2}\left(\left(\omega_{32}+\omega_{33}\right) \Phi^{2}+\omega_{33} \Psi\right)+\omega_{34} \Phi M M^{\prime}+\omega_{35} M^{\prime 2}\right)+O(M),\end{array}\right.$

$a_{t x}=\left\{\begin{array}{l}-\frac{(\gamma-1) Q_{2}}{4 \rho_{0} a_{0}^{2}}+O\left(\varepsilon_{\mathrm{w}}^{0}\right), \\ a_{0}^{2}\left(\omega_{36} M M^{\prime \prime}+M^{2}\left(\left(\omega_{37}+\omega_{38}\right) \Phi^{2}+\omega_{38} \Psi\right)+\omega_{39} \Phi M M^{\prime}+\omega_{40} M^{\prime 2}\right)+O(M),\end{array}\right.$

where $\omega_{1,2, \ldots, 45}$ are constant functions of $\gamma$ given in Table 2, Appendix A.

\section{Appendix C. Derivation of the $Q_{2}$ initial condition}

Here we provide detailed steps towards the arrival of Eq. (4.12), the initial condition of $Q_{2}$ associated with the piston generated shock.

From the kinematic condition of Eq. $(4.7 b)$, the initial jerk of flow between the piston surface and the shock is related to the initial values of partial derivatives $u_{t}, u_{x}, u_{t t}, u_{t x}$ and $u_{x x}$ given in Eqs. (B 1)-(B 4) and (B 5)-(B 8). Particularly in the strong shock limit, the $M^{\prime}$ and $M^{\prime \prime}$ dependency of these derivatives can be expanded using (3.17) and (3.26) to give expressions in terms of $M, Q_{1}, Q_{2}, \Phi$ and $\Psi$. As a result, solving Eq. (4.7b) for $Q_{2}$ and evaluating at $t=0^{+}$gives for $M \rightarrow \infty$,

$$
\begin{aligned}
Q_{2}\left(\beta, 0^{+}\right)=\{- & \zeta_{2}\left[\Phi\left(\iota_{1} M^{2}+\iota_{2} u_{0}^{2}+\iota_{3} u_{0} M\right) M^{3} Q_{1}-\left(\iota_{4} u_{0}^{2}-\iota_{5} M^{2}+\iota_{6} u_{0} M\right) Q_{1}^{2}\right] \\
& -M^{6}\left[\left(\iota_{7} \Phi^{2}+2 \iota_{8} \Psi\right) u_{0} M+\left(\iota_{9} \Phi^{2}+\iota_{10} \Psi\right) M^{2}+\left(\iota_{11} \Phi+\iota_{12} \Psi\right) u_{0}^{2}\right] \\
& \left.+M^{5} \ddot{u}_{0}\right\}_{t=0^{+}} \div\left[\zeta_{2} \gamma_{4} M^{2}\left(\omega_{11} M^{2}+2 \omega_{16} u_{0} M+\omega_{41} u_{0}^{2}\right)\right]_{t=0^{+}},
\end{aligned}
$$

where $\iota_{1,2, \ldots, 12}$ and $\omega_{11,16,41}$ are coefficients given in Table 2, Appendix A. Here the initial 
value of $\Psi$ remains to be determined. This is achieved by substituting the perturbed shock profile (4.4), together with $\Phi\left(\beta, 0^{+}\right)$found in (4.9), into its definition (3.22), implying,

$$
\Psi\left(\beta, 0^{+}\right)=-\frac{\epsilon^{2} \sin ^{2}(\beta)\left(\epsilon^{2} \cos (2 \beta)+2 \epsilon^{2}+1\right)}{\left(\epsilon^{2} \sin ^{2}(\beta)+1\right)^{3}} .
$$

Similarly for the weak shock limit, expanding (4.7b) and solving for $Q_{2}$ directly gives for $M \rightarrow 1$,

$$
\begin{aligned}
Q_{2}\left(\beta, 0^{+}\right)= & \frac{2 \ddot{u}_{0}}{\left(u_{0}-1\right)^{2}}+\left.\frac{4(3 \gamma-1) \Phi(M-1)+(\gamma+1)^{2} Q_{1}}{2(\gamma+1)^{4}\left(u_{0}-1\right)^{2}}\right|_{t=0^{+}}\left\{(\gamma+1)^{2} Q_{1}\right. \\
& \left.\times\left(2(M-1)-u_{0}+1\right)+4 \Phi(M-1)\left[2(\gamma-1)-(3 \gamma-1) u_{0}\right]\right\}_{t=0^{+}} .
\end{aligned}
$$

Now substituting (4.6), (4.8), (4.9) and (C 2) into both of (C 1) and (C 3) prepares the full expression of $Q_{2}\left(\beta, 0^{+}\right)$in terms of the parameters $M_{0}, \epsilon$ and $\ddot{u}_{0}(\beta)$. Setting $\epsilon=0$ then produces

$$
\left.Q_{2}\left(\beta, 0^{+}\right)\right|_{\epsilon=0}= \begin{cases}\frac{M_{0} \ddot{u}_{0}}{\gamma_{4} \zeta_{2}\left(\omega_{11}+2 \alpha_{1} \omega_{16}+\alpha_{1}^{2} \gamma_{4}\right)}, & M_{0} \rightarrow \infty, \\ \frac{2(\gamma+1)^{2} \ddot{u}_{0}}{\left(5+\gamma-4 M_{0}\right)^{2}}, & M_{0} \rightarrow 1,\end{cases}
$$

which must vanish as required by the unperturbed uniform flow condition. Therefore one demands $\ddot{u}_{0}=0$, suggesting that the initial flow attached to the piston has zero acceleration and jerk. Finally, for small $\epsilon$, Eqs. (C 1) and (C 3) are Taylor expanded to give the leading order powers shown in Eq. (4.12),

$$
Q_{2}\left(\beta, 0^{+}\right) \sim \begin{cases}\left(\xi_{1} \cos ^{2}(\beta)+\xi_{2} \sin ^{2}(\beta)\right) M_{0}^{4} \epsilon^{2}, & M_{0} \rightarrow \infty \\ -\frac{32 \gamma \cos ^{2}(\beta)\left(M_{0}-1\right)^{3} \epsilon^{2}}{(\gamma+1)^{3}}, & M_{0} \rightarrow 1\end{cases}
$$

\section{Appendix D. An edge-detection algorithm}

The specific edge-detection algorithm used in Sec. 5 that searches for the onset of a discontinuity on discrete values of the periodic function $M(\beta, t)$ is briefly outlined in this section. The algorithm is taken from a family of spectral methods developed by Gelb \& Tadmor (2000; 2006).

At each time step, discrete data for the Mach number profile, $\bar{M}_{j}$, subject to the rescaling $\min _{j}\left(\bar{M}_{j}\right)=0$ and $\max _{j}\left(\bar{M}_{j}\right)=1$, is obtained first on the size $2 N$ uniform grid $\beta_{j}=2 \pi j /(2 N+1)$, where $j=0,1, \ldots, 2 N$. The discrete concentration detector is defined as

$$
\tilde{T}_{N}^{c}[\bar{M}](\beta)=\pi i \sum_{k=-N}^{N} \operatorname{sgn}(k) c\left(\frac{|k| \Delta \beta}{\pi}\right) \tilde{M}_{k} \exp (i k \beta),
$$

where $\tilde{M}_{k}$ are the Fourier coefficients,

$$
\tilde{M}_{k}=\frac{1}{2 N+1} \sum_{j=0}^{2 N} \bar{M}_{j} \exp \left(-i k \beta_{j}\right)
$$

and $c\left(s_{k}\right)$ with $s_{k}=|k| \Delta \beta / \pi$ is the concentration factor. The following two admissible concentration factors are considered next: the first order polynomial factor,

$$
c^{\mathrm{pol}}\left(s_{k}\right)=\sin \left(\frac{\pi s_{k}}{2}\right) ;
$$


and the exponential factor,

$$
c^{\exp }\left(s_{k}\right)=\sin \left(\frac{\pi s_{k}}{2}\right) \exp \left(\frac{1}{\alpha s_{k}\left(s_{k}-1\right)}\right)\left(\int_{0}^{1} \exp \left(\frac{1}{\alpha s(s-1)}\right) d s\right)^{-1}
$$

where $\alpha=6$ is chosen (Gelb \& Cates 2008). By invoking the minmod operator,

$$
\operatorname{minmod}\left(f_{1}, f_{2}\right)= \begin{cases}\min \left(f_{1}, f_{2}\right), & \text { if } f_{1}, f_{2}>0 \\ \max \left(f_{1}, f_{2}\right), & \text { if } f_{1}, f_{2}<0 \\ 0, & \text { otherwise }\end{cases}
$$

the two concentration factors can be combined to give

$$
\tilde{T}_{N}^{\operatorname{minmod}}[\bar{M}](\beta)=\operatorname{minmod}\left(\tilde{T}_{N}^{\text {pol }}[\bar{M}](\beta), \tilde{T}_{N}^{\exp }[\bar{M}](\beta)\right) .
$$

The concentration factors $c$ are chosen such that all three aforementioned discrete detectors $\tilde{T}_{N}^{c}$ converge to the desired limit that selects jump discontinuities, i.e., $\lim _{\delta \rightarrow 0}[\bar{M}(\beta+\delta)-\bar{M}(\beta-\delta)]$, for all $\beta \in[0,2 \pi)$ as $N \rightarrow \infty$. The separation of scales achieved by these discrete detectors is further enhanced by the nonlinear function,

$$
E_{q, N}=N^{q / 2}\left(\tilde{T}_{N}^{\operatorname{minmod}}[\bar{M}](\beta)\right)^{q},
$$

for some $q>1$. And this leads to the enhanced concentration method

$$
E_{N}^{\operatorname{minmod}}[\bar{M}](\beta)=\left\{\begin{array}{lll}
\tilde{T}_{N}^{\operatorname{minmod}}[\bar{M}](\beta), & \text { if } & \left|E_{q, N}\right|>J_{\text {crit }}, \\
0, & \text { if } & \left|E_{q, N}\right|>J_{\text {crit }}
\end{array}\right.
$$

where $J_{\text {crit }} \sim O(1)$ is a global threshold below which jump discontinuities are neglected. The minmod detector is chosen here for its better performance locating discrete jumps to the nearest grid point while removing spurious oscillations.

\section{REFERENCES}

BAtes, J. W. 2012 On the theory of a shock wave driven by a corrugated piston in a non-ideal fluid. Journal of fluid mechanics 691, 146-164.

BAtes, J. W. 2015 Theory of the corrugation instability of a piston-driven shock wave. Physical Review E 91 (1), 1-6.

Best, J. P. 1991 A generalisation of the theory of geometrical shock dynamics. Shock Waves 1 (4), 251-273.

Best, J. P. 1993 Accounting for transverse flow in the theory of geometrical shock dynamics. Proceedings of the Royal Society of London. Series A: Mathematical and Physical Sciences 442 (1916), 585-598.

Briscoe, M. G. \& Kovitz, A. A. 1968 Experimental and theoretical study of the stability of plane shock waves reflected normally from perturbed flat walls. Journal of Fluid Mechanics 31 (3), 529-546.

Clavin, P. 2013 Nonlinear analysis of shock-vortex interaction: Mach stem formation. Journal of Fluid Mechanics 721, 324-339.

Clavin, P. \& Denet, B. 2002 Diamond patterns in the cellular front of an overdriven detonation. Physical review letters 88 (4), 044502.

Courant, R. \& Friedrichs, K. O. 1999 Supersonic flow and shock waves, , vol. 21. Springer Science \& Business Media.

Denet, B., Biamino, L., Lodato, G., Vervisch, L. \& Clavin, P. 2015 Model equation for the dynamics of wrinkled shockwaves: comparison with dns and experiments. Combustion Science and Technology 187 (1-2), 296-323.

Drake, R. P., Glendinning, S. G., Estabrook, Kent, Remington, B. A., McCray, 
Richard, Wallace, R. J., Suter, L. J., Smith, T. B., Carroll, J. J., London, R. A. \& Liang, E. 1998 Observation of forward shocks and stagnated ejecta driven by high-energy-density plasma flow. Physical Review Letters .

D'yakov, S. P. 1954 Shock wave stability. Zh. Eksp. Teor. Fiz 27 (3), 288-295.

Eggers, J., Grava, T., Herrada, M. A. \& Pitton, G. 2017 Spatial structure of shock formation. Journal of Fluid Mechanics 820, 208-231.

Emanuel, G. 2016 Analytic fluid dynamics, 3rd edn. CRC Press.

Emanuel, G. 2019 Derivatives on the downstream side of a moving, curved shock. Journal of Engineering Mathematics 117 (1), 79-105.

Faria, L. M., Kasimov, A. R. \& Rosales, R. R. 2015 Theory of weakly nonlinear selfsustained detonations. Journal of Fluid Mechanics .

Freeman, N. C. 1955 A theory of the stability of plane shock waves. Proceedings of the Royal Society of London. Series A. Mathematical and Physical Sciences 228 (1174), 341-362.

Freeman, N. C. 1957 On the stability of plane shock waves. Journal of Fluid Mechanics .

Gelb, Anne \& Cates, Dennis 2008 Detection of edges in spectral data iiirefinement of the concentration method. Journal of Scientific Computing 36 (1), 1-43.

Gelb, Anne \& TAdmor, Eitan 2000 Detection of edges in spectral data ii. nonlinear enhancement. SIAM Journal on Numerical Analysis 38 (4), 1389-1408.

Gelb, A. \& TADmor, E. 2006 Adaptive edge detectors for piecewise smooth data based on the minmod limiter. Journal of Scientific Computing 28 (2-3), 279-306.

Hoefer, M. A., Ablowitz, M. J. \& Engels, P. 2008 Piston dispersive shock wave problem. Physical Review Letters .

Hornung, H. G. 2010 Deriving features of reacting hypersonic flow from gradients at a curved shock. AIAA Journal .

KANWAL, R. P. 1957 Determination of the vorticity and the gradients of flow parameters behind a three-dimensional unsteady curved shock wave. Archive for Rational Mechanics and Analysis 1 (1), 225-232.

Katko, B. J., Chavez, R., Liu, H., Lawlor, B., McGuire, C., Zheng, L., Zanteson, J. \& Eliasson, V. 2020 Experimental and numerical study of blast-structure interaction. In Structures Congress 2020, pp. 105-118. American Society of Civil Engineers Reston, VA.

LAPWORTH, K. C. 1959 An experimental investigation of the stability of plane shock waves. Journal of Fluid Mechanics .

LELE, S. K. 1992 Compact finite difference schemes with spectral-like resolution. Journal of computational physics 103 (1), 16-42.

Lighthill, M. J. 1949 The diffraction of blast. i. Proceedings of the Royal Society of London. Series A. Mathematical and Physical Sciences 198 (1055), 454-470.

Lighthill, M. J. 1950 The diffraction of blast. ii. Proceedings of the Royal Society of London. Series A. Mathematical and Physical Sciences 200 (1063), 554-565.

Lindl, J., Landen, O., Edwards, J., Moses, Ed \& team, NIC 2014 Review of the national ignition campaign 2009-2012. Physics of Plasmas 21 (2), 020501.

Lodato, G., Vervisch, L. \& Clavin, P. 2016 Direct numerical simulation of shock wavywall interaction: analysis of cellular shock structures and flow patterns. Journal of Fluid Mechanics 789, 221-258.

Lodato, G., Vervisch, L. \& Clavin, P. 2017 Numerical study of smoothly perturbed shocks in the newtonian limit. Flow, Turbulence and Combustion 99 (3-4), 887-908.

MAJdA, A. \& Rosales, R. 1983 A theory for spontaneous mach stem formation in reacting shock fronts, i. the basic perturbation analysis. SIAM Journal on Applied Mathematics 43 (6), 1310-1334.

Matsui, N., Mima, K., Honda, M. \& Nishiguchi, A. 1999 Analysis of rippled shock-wave propagation and ablation-front stability by theory and hydrodynamic simulation. Journal of Plasma Physics .

Maxwell, A., Dryer, M. \& McIntosh, P. 1985 A piston-driven shock in the solar corona. Solar physics $\mathbf{9 7}$ (2), 401-413.

MöLDER, S. 2016 Curved shock theory. Shock Waves .

Mostert, W, Pullin, D. I., Samtaney, R. \& Wheatley, V. 2017 Geometrical shock dynamics for magnetohydrodynamic fast shocks. Journal of Fluid Mechanics $\mathbf{8 1 1}$. 
Mostert, W., Pullin, D. I., Samtaney, R. \& Wheatley, V. $2018 a$ Singularity formation on perturbed planar shock waves. Journal of Fluid Mechanics 846, 536-562.

Mostert, W., Pullin, D. I., Samtaney, R. \& Wheatley, V. $2018 b$ Spontaneous singularity formation in converging cylindrical shock waves. Physical Review Fluids 3 (7), 071401.

PAnt, J. C. 1969 Some aspects of unsteady curved shock waves. International Journal of Engineering Science .

Strehlow, R. A. \& Fernandes, F. D. 1965 Transverse waves in detonations. Combustion and Flame .

Thomas, T. Y. 1947 On Curved Shock Waves. Journal of Mathematics and Physics .

Van Moorhem, W. K. \& George, A. R. 1975 On the stability of plane shocks. Journal of Fluid Mechanics 68 (1), 97-108.

Wan, Q., Jeon, H., Deiterding, R. \& Eliasson, V. 2017 Numerical and experimental investigation of oblique shock wave reflection off a water wedge. Journal of Fluid Mechanics 826, 732-758.

Whitham, G. B. 1957 A new approach to problems of shock dynamics part i two-dimensional problems. Journal of Fluid Mechanics 2 (2), 145-171.

Whitham, G. B. 2011 Linear and nonlinear waves, , vol. 42. John Wiley \& Sons.

ZAIDEL, R. M. 1967 Development of perturbations in plane shock waves. Journal of Applied Mechanics and Technical Physics . 